\title{
Linguistic Interval-Valued Intuitionistic Fuzzy Copula Heronian Mean Operators for Multiattribute Group Decision-Making
}

\author{
Lei Xu (D), ${ }^{1,2}$ Yi Liu, ${ }^{1,2}$ and Haobin Liu ${ }^{1,2}$ \\ ${ }^{1}$ Data Recovery Key Lab of Sichuan Province, Neijiang Normal University, Neijiang 641000, Sichuan, China \\ ${ }^{2}$ School of Mathematics and Information Science, Neijiang Normal University, Neijiang 641000, Sichuan, China \\ Correspondence should be addressed to Lei Xu; 1986_xulei@163.com
}

Received 3 June 2020; Revised 31 August 2020; Accepted 7 October 2020; Published 19 November 2020

Academic Editor: Harish Garg

Copyright (C) 2020 Lei Xu et al. This is an open access article distributed under the Creative Commons Attribution License, which permits unrestricted use, distribution, and reproduction in any medium, provided the original work is properly cited.

As a generalization of the intuitionistic fuzzy number (IFN), the linguistic interval-valued intuitionistic fuzzy number (LIVIFN) is a flexible and superior tool to describe complex fuzzy uncertainty information. Heronian mean (HM) operator has the characteristic of considering the relationship between attributes. Extended copulas (ECs) and extended cocopulas (ECCs) are the promotion form of Archimedean $t$-norm and $t$-conorm (ATT). ECs and ECCs can generate versatile operational rules and can provide more choice for decision makers (DMs). Therefore, it is very necessary to take advantages of them. In this paper, ECs and ECCs, some specifics of ECs and ECCs, and score and accuracy functions of IVILFNs are gained first. Then, we propose the linguistic interval-valued intuitionistic fuzzy weighted copula Heronian mean (LIVIFWCHM) operator; also, some properties and five specific expressions of the LIVIFWCHM operator are discussed. Moreover, we also propose a new MAGDM approach based on the proposed LIVIFWCHM operator. Finally, a set of examples are used to demonstrate the effectiveness, generality, and flexibility of the proposed method.

\section{Introduction}

Decision-making problems (DMPs) exist in all aspects of people's life, ranging from the development of national politics, economy, and culture to the decision-making at the enterprise level. The multiattribute decision-making (MADM) problem is based on the analysis of multiple influencing factors or indicators in the decision-making. It is necessary to judge or evaluate the limited scheme set from multiple attributes, give the corresponding evaluation information or preference information, and then rank the limited alternatives. With the rapid development of society and the increasingly complex social environment, it is difficult for a decision maker to consider all aspects of the problem, so multiattribute group decision-making (MAGDM) came into being. MAGDM combines the characteristics of MADM and group decision-making. It mainly refers to that many members of the group evaluate several fixed attributes of several alternatives, give the order of alternatives, and obtain the best scheme.
In the classic MADM problems, decision makers (DMs) usually use accurate values to evaluate the attributes of alternatives. However, in the practical application process, due to the complexity and fuzziness of the problem, the evaluation values given by DMs are usually not accurate values but in the form of linguistic values, such as "good," "general," or "poor." Therefore, linguistic-based multiattribute decision-making has become the hot research content of MAGDM [1].

Since Zadeh put forward the concept of the linguistic variable in 1975 [2], the combination of the linguistic variable and other theories has been put forward constantly [3-5], such as linguistic hesitant fuzzy set (LHFS) [6, 7], linguistic neutrosophic set $[8,9]$, linguistic intuitionistic fuzzy set (LIFS) [10], and linguistic Pythagorean fuzzy set [11]. As an important extended linguistic fuzzy set, LIFS has drawn much more attention. Chen et al. [10] first introduced the concept of the LIFS by combining the linguistic term set (LTS) and the IFS in which the membership degree (MD) and nonmembership degree (NMD) are expressed by the 
LTS. A LIFS $A$ on a finite universe of discourse $Z$ and CLTS $\overline{\mathcal{S}}=\left\{s_{\alpha} \mid s_{0} \leq s_{\alpha} \leq s_{t}, \alpha \in[0, t]\right\}$ can be denoted as $A=\left\{\left(s_{\alpha}(z)\right.\right.$, $\left.\left.s_{\beta}(z)\right) \mid z \in Z\right\}$, where $s_{\alpha}(z), s_{\beta}(z) \in \overline{\mathcal{S}}$ with the condition $\alpha+\beta \leq t$.

In order to better express fuzziness, Garg and Kumar [12] introduced the linguistic interval-valued IFS (LIVIFS). LIVIFS is a more general form of the LIFS. For example, selecting a suitable house from a certain number of alternatives is a typical decision problem. In this problem, factors affecting the house selection such as the direction of the house, unit price of area, area size, community environment, and credibility of the developer may be described by linguistic variables (LVs) such as "poor," "medium," and "good." Under this condition, IFS and IVIFS are no longer suitable. Furthermore, in the LIVIFS, the MD and NMD are expressed by the interval LVs (ILVs). When the upper and lower bounds of ILVs are equal, the LIVIFS degenerates to the LIFS. Based on the LIVIFS, scholars have proposed some aggregation operators (AOs), such as a prioritised weighted averaging operator by Kumar and Garg [13], a weighted Maclaurin symmetric mean operator (LIVIFWMSM) by Liu and Qin [14], and a weighted aggregation operator (LIVIFAWPMM) by Qin [15].

There are two limitations among these AOs. Firstly, the aforementioned AOs are only obtained by special $t$-norms (TNs) and $t$-conorms (TCs), i.e., algebraic TN and algebraic TC. Secondly, it is assumed that there is no relationship between attributes. In order to address some of the aforementioned limitations, some improved linguistic intervalvalued intuitionistic fuzzy AOs are developed to solve some DMPs. For example, Xu et al. proposed the interval-valued intuitionistic fuzzy (IVIF) weighted power Muirhead mean (IVIFWPMM) operator [16]; a LIVIF-based Archimedean power Muirhead mean operator by Qin [15], which can tackle DMPs more generally and flexibly; entropic combined weighted averaging operator by Xian et al. [17]; LIVIFSbased Maclaurin symmetric mean (MSM) operator by Liu and Qin [14]; interval-valued intuitionistic 2-tuple linguistic setting and Bonferroni mean by Du and Yuan [18]; Pythagorean fuzzy linguistic (dual) Muirhead mean (PFLMM) operators and their weighted form by Liu [19]; Dombi Heronian mean operators by Wu et al. [20]; and Hamy mean operators by $\mathrm{Wu}$ et al. [21]. Besides, some DMP approaches have been built [22-32].

For the AOs above, although they can do well in their specific circumstances, only a small part of them can provide desirable generality and flexibility while taking into account the correlation between attributes. In [33], Liu and Chen proposed generalized AOs for the intuitionistic 2-tuple linguistic information with three kinds of additive generator. In [34], Tan and Chen proposed generalized AOs for the Archimedean intuitionistic fuzzy information with five kinds of additive generator.

Among various kinds of TNs and TCs, copulas and cocopulas are classical examples of TNs and TCs. Copula [35] can not only reflect the dependence among variables but also prevent information losing in the aggregation process. Copula is a method to deal with the correlation of random variables in statistics. The basic idea of the copula function is to simplify the problem by transforming the marginal variable into a uniformly distributed variable without looking at many different marginal distributions and then define the correlation as a joint distribution on the uniform distribution. As a tool for describing the dependence mechanism between variables, the copula function contains almost all the dependence information of random variables, especially when it is impossible to determine whether the traditional linear correlation coefficient can correctly measure the correlation between variables.

There are two distinguishing features of copulas: (1) copulas and cocopulas are flexible because DMs can select different types of copulas to define the operations under the fuzzy environment, and the results obtained from these operations are close; (2) copula function is flexible to capture the correlations among attributes in DMPs. Based on the two obvious characteristics, copulas have been applied to some DMPs. For example, Nelsen [35] applied copulas in the aggregation function. Tao et al. [36] extended copulas to the IFS and applied it to DMPs. Basically, there are two types of copula: Archimedean copula and Gaussian copula. In this paper, we only discuss Archimedean copula. In the light of Archimedean copula, Tao et al. [37] studied a new computational model for unbalanced LVs. Chen et al. [38] defined new AOs in the linguistic neutrosophic set based on the copula and applied them to solve DMPs. Xu et al. [39] also proposed fuzzy copula power AOs to solve MAGDM problems based on linguistic interval-valued intuitionistic information; however, the authors did not consider the correlation between attributes.

In order to solve the relationship between attributes, Bonferroni [40] proposed the Bonferroni mean (BM) operator firstly, and then Yager [41] further expanded the BM operator and enhanced its modeling capabilities. By replacing simple average operators (ordered weighted average operators and Choquet operators) with other forms of average operators, Yager proposed some more efficient AOs. However, the BM operator ignores the relationship between each attribute and itself and with the nature of computation redundancy. Heronian mean (HM) operator was first proposed by Beliakov [42]. Heronian mean (HM) operator and BM operator have similar structures, and both consider the correlation between attribute values. However, the HM operator has obvious advantages over the BM operator and can make up for the two shortcomings of the BM operator. On this basis, a series of extension models have been proposed, such as the intuitionistic fuzzy geometric HM (IFGHM) operator [43], IVIF Heronian mean (IVIFHM) operator [44], uncertain linguistic HM operators [45], partitioned HM operators [46], unbalanced linguistic generalized HM operator [32], normal intuitionistic fuzzy HM operator [47], and picture fuzzy Dombi HM operator [48]. However, the HM is not applied to aggregate the linguistic interval-valued intuitionistic fuzzy information (LIVIFI).

Although the existing AOs can provide the most commonly used way to aggregate the LIVIFS, they lack a unique way in practical applications. What is it the form of AOs on the basis of the copula function and LIVIFI? What are the differences between copula-based AOs and existing AOs? 
Considering the HM operator has the ability to interrelate among the attributes, what is the form of the weighted HM operator based on the LIVIFS and copula function? So, the goal and motivation of the present work are to synthesize ECs (ECCs), HM operator, and LIVIFS and to develop a MAGDM approach with LIVIFI.

Accordingly, the main intentions and contributions of this work are summarized as follows:

(1) We propose a new version of copulas and cocopulas by extending the domain and the range of copulas and cocopulas from $[0,1]$ to $[0, t](t>0)$, which is called extended copulas (ECs) and extended cocopulas (ECCs)

(2) We introduce several universal operational laws of LIVIFNs and discuss some special instances

(3) We develop the LIVIFWCHM operator, explore several characteristics, and give some particular cases

(4) In addition, we propose a novel decision approach for MAGDM with LIVIFI and investigate the efficacy and superiorities of the propounded approach

In order to achieve the above goals, the organizational structure of this paper is as follows. In Section 2, some basic concepts of the LIVIFS, copulas, and cocopulas and some properties of the LIVIFS based on ECs and ECCs are introduced. Furthermore, we redefine several novel operations for LIVIFNs and discuss some special cases. In Section 3, based on these operation rules, we derive the LIVIFWCHM operator as well as explore several properties and particular examples. In Section 4, a new method for MAGDM is proposed based on the LIVIFWCHM operator under LIVIFI. In Section 5, a set of examples are provided to investigate the efficacy and superiority of the propounded approach. The conclusion is obtained in Section 6.

\section{Preliminaries}

In this section, firstly, some basic concepts related to the LIVIFS, HM operator, and copulas and cocopulas are reviewed, which are the basis of the present work.

Definition 1 (see [12]). Let $X$ be a finite universal set and $S_{[0, t]}$ be a continuous LTS. A LIVIFS $A$ in $X$ is defined as

$$
A=\left(x, s_{\mu_{A}(x)}, s_{\nu_{A}(x)}\right), \quad \mid x \in X,
$$

where $s_{\mu A(x)}=\left[s_{\mu_{A}^{L}(x)}, s_{\mu_{A}^{U}(x)}\right]$ and $s_{v A(x)}=\left[s_{v_{A}^{L}(x)}, s_{v_{A}^{U}(x)}\right]$ are all subsets of $\left[s_{0}, s_{t}\right]$ and represent linguistic MD and NMD of $x$ to $A$, respectively. For any $x \in X, s_{\mu_{A}^{U}(x)}+s_{\gamma_{A}^{U}(x)} \leq s_{t}$. The pair $\left(\left[s_{\mu_{A}^{L}}, s_{\mu_{A}^{U}}\right],\left[s_{\gamma_{A}^{L}}, s_{\gamma_{A}^{U}}\right]\right)$ is called the LIVIFN.

For convenience, we denote the LIVAIFN as $\alpha=$ $\left(\left[s_{a}, s_{b}\right],\left[s_{c}, s_{d}\right]\right)$, where $s_{a}, s_{b}, s_{c}, s_{d} \in S_{[0, t]}$, and also, $\left[s_{a}, s_{b}\right] \in\left[s_{0}, s_{t}\right],\left[s_{c}, s_{d}\right] \in\left[s_{0}, s_{t}\right], b+d \leq t$.

Definition 2 (see [12]). Let $\alpha=\left(\left[s_{a}, s_{b}\right],\left[s_{c}, s_{d}\right]\right)$ be a LIVAIFN; a score function and accuracy function of $\alpha$ are defined as

$$
\begin{aligned}
S(\alpha) & =s_{(2 t+a-c+b-d) / 4}, \\
H(\alpha) & =s_{(a+b+c+d) / 2} .
\end{aligned}
$$

Then, for any two different LIVAIFNs $\alpha_{1}$ and $\alpha_{2}$, we have the following:

(1) If $S\left(\alpha_{1}\right)<S\left(\alpha_{2}\right)$, then $\alpha_{1}<\alpha_{2}$

(2) If $S\left(\alpha_{1}\right)=S\left(\alpha_{2}\right)$ and $H\left(\alpha_{1}\right)=H\left(\alpha_{2}\right)$, then $\alpha_{1}<\alpha_{2}$

Definition 3 (see [33]). An extended $t$-norm $\mathscr{T}$ is a mapping from $[0, t]^{2}$ to $[0, t]$ if $\mathscr{T}$ fulfills the following: for all $c, d, e \in[0, t]$,

(i) $\mathscr{T}(c, t)=c$.

(ii) $\mathscr{T}(c, d)=\mathscr{T}(d, c)$.

(iii) $\mathscr{T}(c, \mathscr{T}(d, e))=\mathscr{T}(\mathscr{T}(c, d), e)$.

If $T$ just satisfies (T1), then $T$ is called a semicopula. With the help of extended TNs and extended TCs, we first introduce the concept of extended copulas (ECs) and extended cocopulas (ECCs) in order to handle some DMPs with LIFI.

Definition 4 (see [35]). A binary function $\mathbb{C}:[0, t]^{2} \longrightarrow$ $[0, t]$ is called an EC if $\mathbb{C}$ fulfills the following conditions: for all $c, d, c^{\prime}, d^{\prime} \in[0, t]$,

(i) $\mathbb{C}(c, d)+\mathbb{C}\left(c^{\prime}, d^{\prime}\right) \geq \mathbb{C}\left(c, d^{\prime}\right)+\mathbb{C}\left(c^{\prime}, d\right)$.

(ii) $\mathbb{C}(c, 0)=\mathbb{C}(0, c)=0$.

(iii) $\mathbb{C}(c, t)=\mathbb{C}(t, c)=c$.

Definition 5 (see [35]). Let $\rangle:[0, t] \longrightarrow[0,+\infty)$ and $\psi:[0,+\infty) \longrightarrow[0, t]$. If $\varrho, \psi$ satisfy the following conditions, for all $(c, d) \in[0, t]^{2}$,

(1) $\varrho$ is continuous.

(2) $\varrho$ is strictly decreasing.

(3) $\varrho(t)=0$.

(4)

$$
\begin{aligned}
\psi(c) & = \begin{cases}\varrho^{-1}(c), & c \in[0, \varrho(0)], \\
0, & c \in[\varrho(0),+\infty),\end{cases} \\
\mathbb{C}(c, d) & =\psi(\varrho(c)+\varrho(d)),
\end{aligned}
$$

the copula $\mathbb{C}$ is called ECs.

The generator $\varrho$ of an EC is if a mapping from $[0, t]$ to $\mathbf{R}^{+}$ and $\varrho^{-1}$ is the mapping from $\mathbf{R}^{+}$to $[0, t]$ with $\varrho(0)=+\infty$ and $\varrho(t)=0$. According to Genest and Mackay [49], $\mathbb{C}$ can be rewritten as

$$
\mathbb{C}(c, d)=\varrho^{-1}(\varrho(c)+\varrho(d)) .
$$

Definition 6. Let $\mathbb{C}$ be an EC, for all $(c, d) \in[0, t]^{2}$; then, ECCs are expressed as

$$
\mathbb{C}^{*}(c, d)=t-\mathbb{C}(t-c, t-d) .
$$


Theorem 1. For all $c_{1}, c_{2}, d_{1}, d_{2} \in[0, t]$, if $c_{i}+d_{i} \leq$ $t(i=1,2)$, then $0 \leq \mathbb{C}\left(c_{1}, c_{2}\right)+\mathbb{C}^{*}\left(d_{1}, d_{2}\right) \leq t$.

Proof. It follows easily from the definitions of EC and ECC that $0 \leq \mathbb{C}\left(c_{1}, c_{2}\right)+\mathbb{C}^{*}\left(d_{1}, d_{2}\right)$. So, we just need to prove $\mathbb{C}\left(c_{1}, c_{2}\right)+\mathbb{C}^{*}\left(d_{1}, d_{2}\right) \leq t$.

It follows from the definitions of EC and ECC that

$$
\begin{aligned}
\mathbb{C}\left(c_{1}, c_{2}\right)+\mathbb{C}^{*}\left(d_{1}, d_{2}\right)= & \mathbb{C}\left(c_{1}, c_{2}\right)+\left(t-\mathbb{C}\left(t-d_{1}, t-d_{2}\right)\right) \\
= & \left(\varrho^{-1}\left(\varrho\left(c_{1}\right)+\varrho\left(c_{2}\right)\right)\right) \\
& +t-\left(\varrho^{-1}\left(\varrho\left(t-d_{1}\right)+\varrho\left(1-d_{2}\right)\right)\right) .
\end{aligned}
$$

As $\varrho$ is strictly decreasing and $c_{i}+d_{i} \leq t(i=1,2)$, it follows that

$$
\varrho\left(c_{1}\right)+\varrho\left(c_{2}\right) \geq \varrho\left(t-d_{1}\right)+\varrho\left(t-d_{1}\right) .
$$

Therefore,

$$
\varrho^{-1}\left(\varrho\left(c_{1}\right)+\varrho\left(c_{2}\right)\right) \leq \varrho^{-1}\left(\varrho\left(t-d_{1}\right)+\varrho\left(t-d_{1}\right)\right) .
$$

So, we have

$$
\begin{aligned}
\mathbb{C}\left(c_{1}, c_{2}\right)+\mathbb{C}^{*}\left(d_{1}, d_{2}\right)= & \left(\varrho^{-1}\left(\varrho\left(c_{1}\right)+\varrho\left(c_{2}\right)\right)\right) \\
& +t-\varrho^{-1}\left(\varrho\left(t-d_{1}\right)+\varrho\left(t-d_{2}\right)\right) \\
\leq & \left(\varrho^{-1}\left(\varrho\left(c_{1}\right)+\varrho\left(c_{2}\right)\right)\right) \\
& +t-\left(\varrho^{-1}\left(\varrho\left(c_{1}\right)+\varrho\left(c_{2}\right)\right)\right)=t .
\end{aligned}
$$

According to Theorem 1, we know that the operation of ECs and ECCs is close. Table 1 shows five common Archimedean copulas, which can be considered for further consideration. In the following, we will give a new version of operational rules based on ECs and ECCs.

Definition 7. Let $\alpha_{1}=\left(\left[s_{a_{1}}, s_{b_{1}}\right],\left[s_{c_{1}}, s_{d_{1}}\right]\right)$ and $\alpha_{2}=\left(\left[s_{a_{2}}, s_{b_{2}}\right]\right.$, $\left.\left[s_{c_{2}}, s_{d_{2}}\right]\right)$ be two LIVIFNs; the novel operational rules of LIVIFNs are given as follows:

$$
\begin{aligned}
(L 1) \alpha_{1} \oplus_{\mathbb{C}} \alpha_{2} & =\left(\left[s_{t-\left(\varrho^{-1}\left(\varrho\left(t-a_{1}\right)+\varrho\left(t-a_{2}\right)\right)\right)}, s_{t-\left(\varrho^{-1}\left(\varrho\left(t-b_{1}\right)+\varrho\left(t-b_{2}\right)\right)\right)}\right],\left[s_{\varrho^{-1}}\left(\varrho\left(c_{1}\right)+\varrho\left(c_{2}\right)\right), s_{\varrho^{-1}}\left(\varrho\left(d_{1}\right)+\varrho\left(d_{2}\right)\right)\right]\right), \\
(L 2) \alpha_{1} \otimes_{\mathbb{C}} \alpha_{2} & =\left(\left[s_{\varrho^{-1}\left(\varrho\left(a_{1}\right)+\varrho\left(a_{2}\right)\right)}, s_{\varrho^{-1}}\left(\varrho\left(b_{1}\right)+\varrho\left(b_{2}\right)\right)\right],\left[s_{t-\left(\varrho^{-1}\left(\varrho\left(t-c_{1}\right)+\varrho\left(t-c_{2}\right)\right)\right),} s_{\left.\left.t-\left(\varrho^{-1}\left(\varrho\left(t-d_{1}\right)+\varrho\left(t-d_{2}\right)\right)\right)\right]\right) .}\right.\right.
\end{aligned}
$$

It is easy to verify that $\oplus_{\mathbb{C}}$ and $\otimes_{\mathbb{C}}$ satisfy the associative law, that is, for all three LVIIFNs $A, B$, and $C$,

$$
\begin{aligned}
(1)\left(A \oplus_{\mathbb{C}} B\right) \oplus_{\mathbb{C}} C & =A \oplus_{\mathbb{C}}\left(B \oplus_{\mathbb{C}} C\right), \\
(2)\left(A \otimes_{\mathbb{C}} B\right) \otimes_{\mathbb{C}} C & =A \otimes_{\mathbb{C}}\left(B \otimes_{\mathbb{C}} C\right) .
\end{aligned}
$$

Theorem 2. Let $\alpha=\left(\left[s_{a}, s_{b}\right],\left[s_{c}, s_{d}\right]\right)$ be a LIVIFN; for $n \in \mathbf{N}^{*}$, we have $n \alpha$ is still a LIVAIFN, and

$$
\begin{aligned}
& n \alpha=\left(\left[s_{t-\left(\varrho^{-1}(n \varrho(t-a))\right)}, s_{t-\left(\varrho^{-1}(n \varrho(t-b))\right)}\right],\right. \\
& {\left.\left[s_{\varrho^{-1}(n \varrho(c))}, s_{\varrho^{-1}(n \varrho(d))}\right]\right), } \\
& n
\end{aligned}
$$

where $n \alpha=\overbrace{\alpha \oplus_{\mathbb{C}} \alpha \oplus_{\mathbb{C}} \cdots \oplus_{\mathbb{C}} \alpha}^{n}$.
Proof. It is easy to obtain from Theorem 1 that $n \alpha$ is a LIVAIFN. Now, we only prove that equation (13) holds for $n \in \mathbf{N}^{*}$. When $n=1$,

$$
\begin{aligned}
1 \alpha & =\left(\left[s_{t-\left(\varrho^{-1}(\varrho(t-a))\right)}, s_{t-\left(\varrho^{-1}(\varrho(t-b))\right)}\right],\left[s_{\varrho^{-1}(\varrho(c))}, s_{\varrho^{-1}(\varrho(d))}\right]\right) \\
& =\left(\left[s_{a}, s_{b}\right],\left[s_{c}, s_{d}\right]\right)=\alpha .
\end{aligned}
$$

Presume equation (13) holds for $n=k$, i. e.,

$$
k \alpha=\left(\left[s_{t-\left(\varrho^{-1}(k \varrho(t-a))\right)}, s_{t-\left(\varrho^{-1}(k \varrho(t-b))\right)}\right],\left[s_{\varrho^{-1}(k \varrho(c))}, s_{\varrho^{-1}(k \varrho(d))}\right]\right) .
$$

When $n=k+1$, we have

$$
\begin{aligned}
& (k+1) \alpha=k \alpha \oplus_{\mathbb{C}} \alpha \\
& =\left(\left[s_{t-\left(\varrho^{-1}(k \varrho(t-a))\right)}, s_{t-\left(\varrho^{-1}(k \varrho(t-b))\right)}\right],\left[s_{\varrho^{-1}(k \varrho(c))}, s_{\varrho^{-1}(k \varrho(d))}\right]\right) \oplus_{\mathbb{C}}\left(\left[s_{a}, s_{b}\right],\left[s_{c}, s_{d}\right]\right) \\
& =\left(\left[s_{t-\left(\varrho^{-1}\left(\varrho\left(t-\left(t-\left(\varrho^{-1}(k \varrho(t-a))\right)\right)\right)\right)+\varrho(t-a)\right)}, s_{t-\left(\varrho^{-1}\left(\varrho\left(t-\left(t-\left(\varrho^{-1}(k \varrho(t-b))\right)\right)\right)\right)+\varrho(t-b)\right)}\right],\left[s_{\varrho^{-1}(k \varrho(c)+\varrho(c))}, s_{\varrho^{-1}(k \varrho(d)+\varrho(d))}\right]\right) \\
& =\left(\left[s_{t-\left(\varrho^{-1}((k \varrho(t-a))+\varrho(t-a))\right)}, s_{t-\left(\varrho^{-1}((k \varrho(t-b))+\varrho(t-b))\right)}\right],\left[s_{\varrho^{-1}(k \varrho(c)+\varrho(c))}, s_{\varrho^{-1}(k \varrho(d)+\varrho(d))}\right]\right) \\
& =\left(\left[s_{t-\left(\varrho^{-1}((k+1) \varrho(t-a))\right)}, s_{t-\left(\varrho^{-1}((k+1) \varrho(t-b))\right)}\right],\left[s_{\varrho^{-1}((k+1) \varrho(c))}, s_{\varrho^{-1}((k+1) \varrho(d))}\right]\right) \text {. }
\end{aligned}
$$


TABLE 1: The influence of parameter $\theta$ on the rank of alternatives.

\begin{tabular}{|c|c|c|c|}
\hline Type & Generator $\varrho(c)$ & $\mathrm{EC}$ and ECC & Condition \\
\hline Gumbel & $\varrho(c)=(-\ln (c / t))^{\theta}$ & $\begin{array}{c}\mathbb{C}(c, d)=\mathrm{te}^{-\left((-\ln (c / t))^{\theta}+(-\ln (d / t))^{\theta}\right)^{(1 / \theta)}} \\
\mathbb{C}^{*}(c, d)=t-\mathrm{te}^{-\left((-\ln (t-c / t))^{\theta}+(-\ln (t-d / t))^{\theta}\right)^{(1 / \theta)}}\end{array}$ & $\theta \geq 1$ \\
\hline Clayton & $\varrho(c)=(c / t)^{-\theta}-1$ & $\begin{array}{c}\mathbb{C}(c, d)=t\left((c / t)^{-\theta}+(d / t)^{-\theta}-1\right)^{-(1 / \theta)} \\
\mathbb{C}^{*}(c, d)=t-t\left((t-c / t)^{-\theta}+(t-d / t)^{-\theta}-1\right)^{-(1 / \theta)}\end{array}$ & $\theta \neq 0$ \\
\hline Frank & $\varrho(c)=\ln \left(e^{-(\theta c / t)}-1 / e^{-\theta}-1\right)$ & $\begin{array}{c}\mathbb{C}(c, d)=(-(t / \theta)) \ln \left[\left(\left(e^{-(\theta c / t)}-1\right)\left(e^{-(\theta d / t)}-1\right) / e^{-\theta}-1\right)+1\right] \\
\mathbb{C}^{*}(c, d)=t+(t / \theta) \ln \left[\left(\left(e^{-(\theta(t-c) / t)}-1\right)\left(e^{-(\theta(t-d) / t)}-1\right) / e^{-\theta}-1\right)+1\right]\end{array}$ & $\theta \neq 0$ \\
\hline Ali-Mikhail-Haq & $\varrho(c)=\ln (t-\theta(t-c) / c)$ & $\begin{array}{c}\mathbb{C}(c, d)=\left(\mathrm{tcd} / t^{2}-\theta(t-c)(t-d)\right) \\
\mathbb{C}^{*}(c, d)=t-\left(t(t-c)(t-d) / t^{2}-\theta c d\right)\end{array}$ & $\theta \in[-1,1)$ \\
\hline Joe & $\varrho(c)=-\ln \left(1-(1-(c / t))^{\theta}\right)$ & $\begin{array}{c}\mathbb{C}(c, d)=t-\left(\left(t^{\theta}\left((t-c)^{\theta}+(t-d)^{\theta}\right)-(t-c)^{\theta}(t-d)^{\theta}\right)^{(1 / \theta)} / t\right) \\
\mathbb{C}^{*}(c, d)=t\left(c^{\theta}+d^{\theta}-(\mathrm{cd} / t)^{\theta}\right)^{(1 / \theta)}\end{array}$ & $\theta \geq 1$ \\
\hline
\end{tabular}

So, equation (13) holds for all $n \in \mathbf{N}^{*}$.

Similarly, the following theorem can be obtained easily.
Theorem 3. Let $\alpha=\left(\left[s_{a}, s_{b}\right],\left[s_{c}, s_{d}\right]\right)$ be a LIVIFN; for all $n \in \mathbf{N}^{*}$, we have $\alpha^{n}$ is still a LIVIFN, and
It is easy to verify that the operational laws hold, for all three LIVIFNs $\alpha, \alpha_{1}, \alpha_{2}$ and $\lambda, \lambda_{1}, \lambda_{2}>0$ :

$$
\begin{aligned}
& \text { (3) } \lambda_{1} \alpha \oplus_{\mathbb{C}} \lambda_{2} \alpha=\left(\lambda_{1}+\lambda_{2}\right) \alpha, \\
& \text { (4) } \alpha_{1}^{\lambda} \otimes_{\mathbb{C}} \alpha_{2}^{\lambda}=\left(\alpha_{1} \otimes_{\mathbb{C}} \alpha_{2}\right)^{\lambda}, \\
& \text { (5) } \alpha_{1}^{\lambda} \otimes_{\mathbb{C}} \alpha_{2}^{\lambda}=\alpha^{\lambda_{1}+\lambda_{2}} .
\end{aligned}
$$

Definition 8 (see [43]). Let $I=[0, t], p, q \geq 0, H^{p, q}: I^{n} \longrightarrow I$ if $H^{p, q}$ satisfies

$$
H^{p, q}\left(x_{1}, \ldots, x_{n}\right)=\left(\frac{2}{n(n+1)} \sum_{i=1}^{n} \sum_{j=1}^{i} x_{i}^{p} x_{j}^{q}\right)^{(1 / p+q)} .
$$

\section{LIVIF Heronian Mean Operator Based on ECs and ECCs}

Under this part, we proposed the LIVIFWCHM operator through the novel operational laws. The particular cases of the propounded operator are explored, and several desired properties are proved in detail.

Definition 9. Let $\alpha_{i}=\left(\left[s_{a_{i}}, s_{b_{i}}\right],\left[s_{c_{i}}, s_{d_{i}}\right]\right)$ be a collection of LIVIFNs and $p, q>0$; then, the linguistic interval-valued intuitionistic fuzzy weight copula Heronian mean (LIVIFWCHM) operator is expressed as

$$
\operatorname{LIVIFWCHM}^{p, q}\left(\alpha_{1}, \ldots, \alpha_{n}\right)=\left(\frac{2}{n(n+1)} \sum_{i=1}^{n} \sum_{j=1}^{i}\left(\omega_{i} \alpha_{i}\right)^{p} \otimes\left(\omega_{j} \alpha_{j}\right)^{q}\right)^{(1 / p+q)}
$$

where $\omega_{i}$ is the weight vector (WV) of $\alpha_{i}, \omega_{i} \geq 0$, and $\sum_{k=1}^{n} \omega_{i}=1$.
Theorem 4. Let $\alpha_{i}=\left(\left[s_{a_{i}}, s_{b_{i}}\right],\left[s_{c_{i}}, s_{d_{i}}\right]\right)$ be a collection of LIVIFNs and $p, q>0$; then, the aggregated result form is still LIVIFNs and has 
$\operatorname{LIVIFWCHM}^{p, q}\left(\alpha_{1}, \ldots, \alpha_{n}\right)=\left(\left[s_{a}, s_{b}\right],\left[s_{c}, s_{d}\right]\right)$,

$$
\begin{aligned}
& a=\varrho^{-1}\left(\frac{1}{(p+q)} \varrho\left(t-\varrho^{-1}\left(\frac{2}{n(n+1)} \sum_{i=1}^{n} \sum_{j=1}^{i} \varrho\left(t-\varrho^{-1}\left(p \varrho\left(t-\left(\varrho^{-1}\left(\omega_{i} \varrho\left(t-a_{i}\right)\right)\right)\right)+q \varrho\left(t-\left(\varrho^{-1}\left(\omega_{j} \varrho\left(t-a_{j}\right)\right)\right)\right)\right)\right)\right)\right)\right), \\
& b=\varrho^{-1}\left(\frac{1}{(p+q)} \varrho\left(t-\varrho^{-1}\left(\frac{2}{n(n+1)} \sum_{i=1}^{n} \sum_{j=1}^{i} \varrho\left(t-\varrho^{-1}\left(p \varrho\left(t-\left(\varrho^{-1}\left(\omega_{i} \varrho\left(t-b_{i}\right)\right)\right)\right)+q \varrho\left(t-\left(\varrho^{-1}\left(\omega_{j} \varrho\left(t-b_{j}\right)\right)\right)\right)\right)\right)\right)\right)\right) \\
& c=t-\left(\varrho^{-1}\left(\frac{1}{(p+q)} \varrho\left(t-\varrho^{-1}\left(\frac{2}{n(n+1)} \sum_{i=1}^{n} \sum_{j=1}^{i} \varrho\left(t-\varrho^{-1}\left(p \varrho\left(t-\varrho^{-1}\left(\omega_{i} \varrho\left(c_{i}\right)\right)\right)+q \varrho\left(t-\varrho^{-1}\left(\omega_{j} \varrho\left(c_{j}\right)\right)\right)\right)\right)\right)\right)\right)\right), \\
& d=t-\left(\varrho^{-1}\left(\frac{1}{(p+q)} \varrho\left(t-\varrho^{-1}\left(\frac{2}{n(n+1)} \sum_{i=1}^{n} \sum_{j=1}^{i} \varrho\left(t-\varrho^{-1}\left(p \varrho\left(t-\varrho^{-1}\left(\omega_{i} \varrho\left(d_{i}\right)\right)\right)+q \varrho\left(t-\varrho^{-1}\left(\omega_{j} \varrho\left(d_{j}\right)\right)\right)\right)\right)\right)\right)\right)\right)
\end{aligned}
$$

Proof. Since

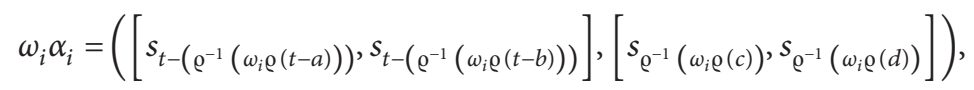

$$
\begin{aligned}
& \left(\omega_{i} \alpha_{i}\right)^{p}=\left(\left[s_{\varrho^{-1}}\left(p \varrho\left(t-\left(\varrho^{-1}\left(\omega_{i} \varrho\left(t-a_{i}\right)\right)\right)\right)\right), s_{\varrho^{-1}}\left(p \varrho\left(t-\left(\varrho^{-1}\left(\omega_{i} \varrho\left(t-a_{i}\right)\right)\right)\right)\right)\right],\left[s_{t-\varrho^{-1}}\left(p \varrho\left(t-\varrho^{-1}\left(\omega_{i} \varrho\left(c_{i}\right)\right)\right)\right), s_{t-\varrho^{-1}}\left(p \varrho\left(t-\varrho^{-1}\left(\omega_{i} \varrho\left(d_{i}\right)\right)\right)\right)\right]\right),
\end{aligned}
$$

and similarly, we have

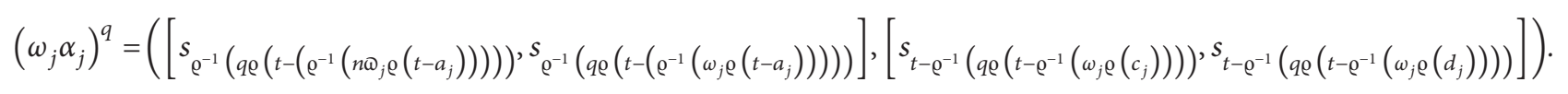

Then,

$$
\begin{aligned}
& \left(\omega_{i} \alpha_{i}\right)^{p} \otimes\left(\omega_{j} \alpha_{j}\right)^{q}
\end{aligned}
$$

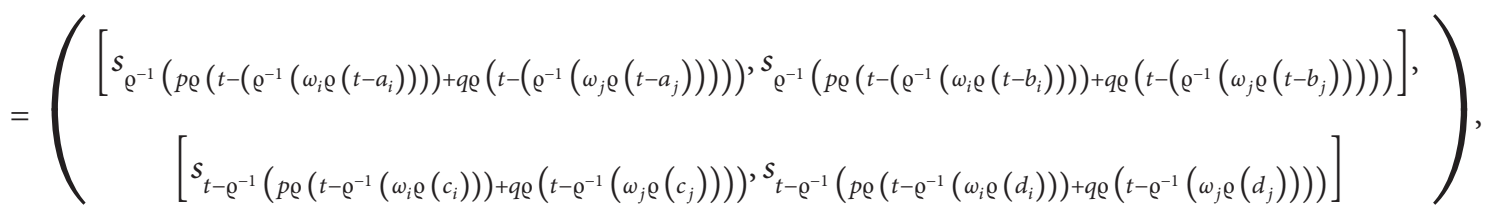


Journal of Mathematics

7

$$
\begin{aligned}
& \sum_{i=1}^{n} \sum_{j=1}^{i}\left(\omega_{i} \alpha_{i}\right)^{p} \otimes\left(\omega_{j} \alpha_{j}\right)^{q}
\end{aligned}
$$

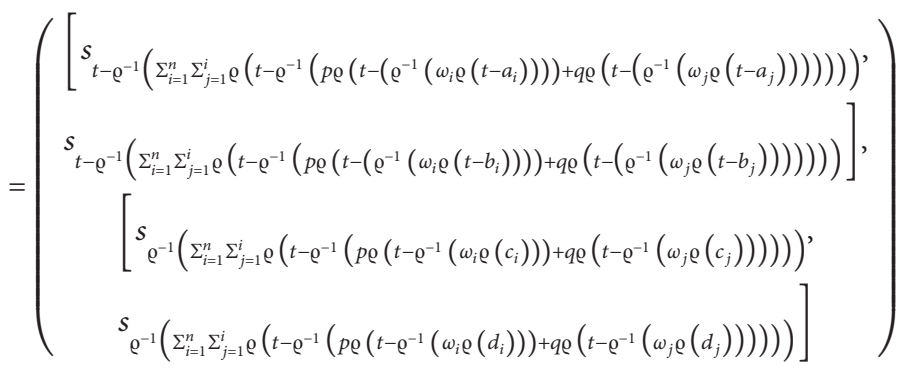

$$
\begin{aligned}
& \frac{2}{n(n+1)} \sum_{i=1}^{n} \sum_{j=1}^{i}\left(\omega_{i} \alpha_{i}\right)^{p} \otimes\left(\omega_{j} \alpha_{j}\right)^{q}
\end{aligned}
$$

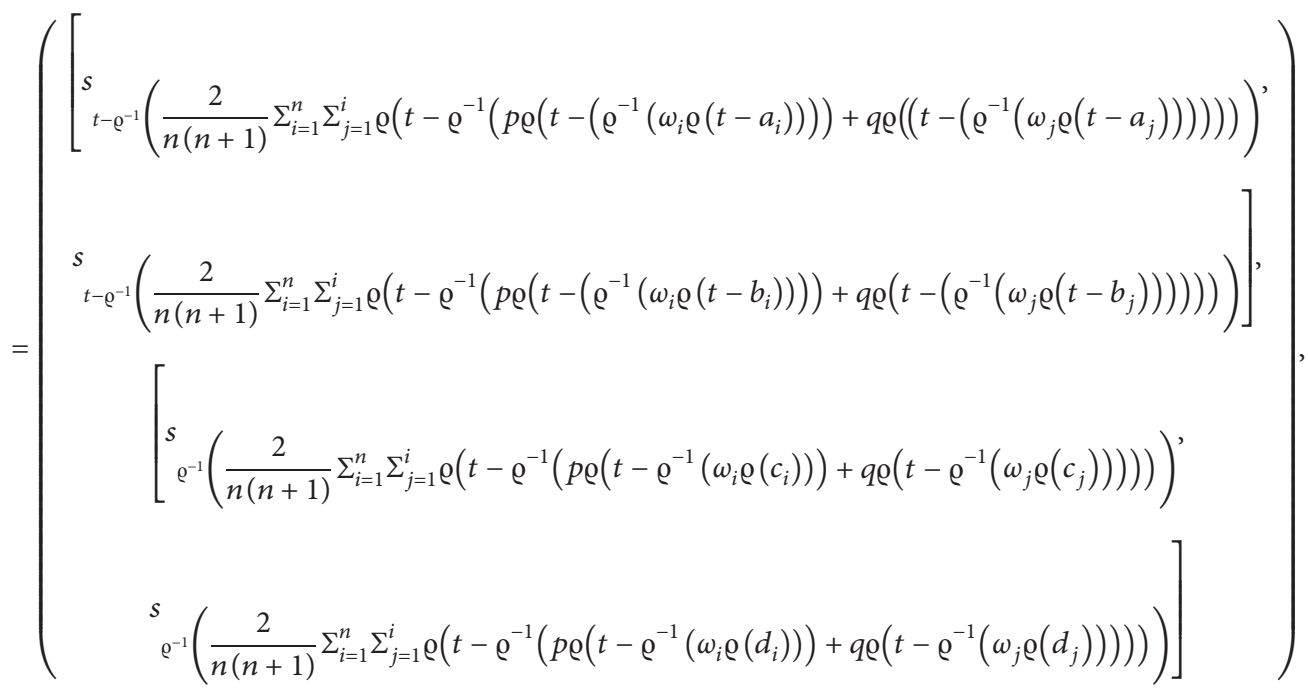

$$
\begin{aligned}
& \left(\frac{2}{n(n+1)} \sum_{i=1}^{n} \sum_{j=1}^{i}\left(\omega_{i} \alpha_{i}\right)^{p} \otimes\left(\omega_{j} \alpha_{j}\right)^{q}\right)^{(1 /(p+q))}
\end{aligned}
$$

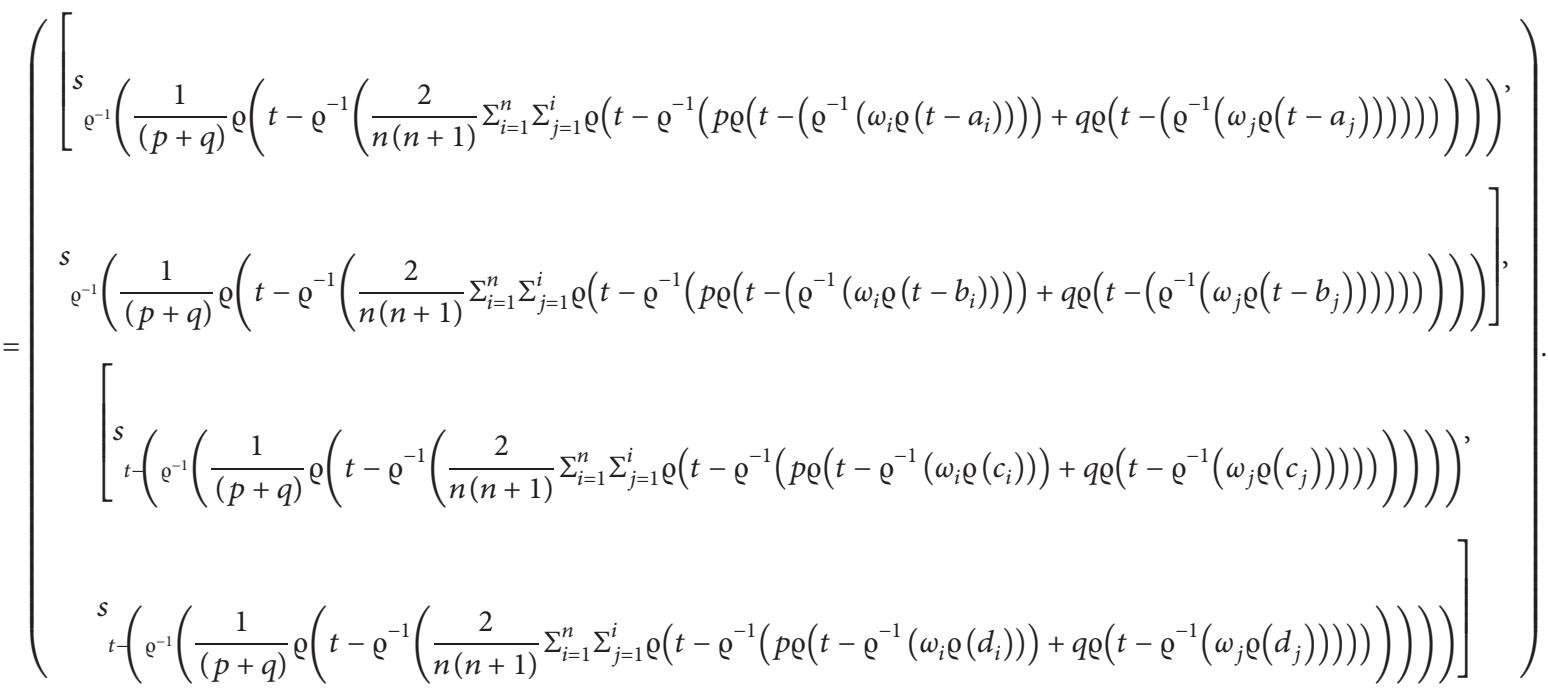

(25) 
Theorem 5 (monotonicity). Let $\alpha_{i}=\left(\left[s_{a_{i}}, s_{b_{i}}\right],\left[s_{c_{i}}, s_{d_{i}}\right]\right)$ and $\beta_{i}=\left(\left[s_{\tau_{i}}, s_{\theta_{i}}\right],\left[s_{\eta_{i}}, s_{v_{i}}\right]\right)$ be a collection of LIVIFNs; if $a_{i} \leq \tau_{i}, b_{i} \leq \theta_{i}, c_{i} \geq \eta_{i}, d_{i} \geq v_{i}$ for all $i$, then

$\operatorname{LIVIFWCHM}^{p, q}\left(\alpha_{1}, \ldots, \alpha_{n}\right) \leq \operatorname{LIVIFWCHM}^{p, q}\left(\beta_{1}, \ldots, \beta_{n}\right)$.
Proof. On the one hand, since $a_{i} \leq \tau_{i}, b_{i} \leq \theta_{i}, c_{i} \geq \eta_{i}, d_{i} \geq v_{i}$ for all $i$, we have $t-a_{i} \geq t-\tau_{i}$ and $t-b_{i} \geq t-\theta_{i}$. As $\varrho$ and $\varrho^{-1}$ are monotonicity decreasing, $\varrho\left(t-a_{i}\right) \leq \varrho\left(t-\tau_{i}\right)$ and $\varrho\left(t-b_{i}\right) \leq \varrho\left(t-\theta_{i}\right)$; furthermore,

$$
\left(\varrho^{-1}\left(w_{i} \varrho\left(t-a_{i}\right)\right)\right) \geq\left(\varrho^{-1}\left(w_{i} \varrho\left(t-\tau_{i}\right)\right)\right),
$$

and so,

$$
\begin{aligned}
& t-\left(\mathrm{e}^{-1}\left(w_{i} \mathrm{e}\left(t-a_{i}\right)\right)\right) \leq t-\left(\mathrm{e}^{-1}\left(w_{i} \mathrm{e}\left(t-\tau_{i}\right)\right)\right) \\
& p \mathrm{e}\left(t-\left(\mathrm{e}^{-1}\left(w_{i} \mathrm{e}\left(t-a_{i}\right)\right)\right)\right) \geq \mathrm{p} \mathrm{\varrho}\left(t-\left(\mathrm{e}^{-1}\left(w_{i} \mathrm{e}\left(t-\tau_{i}\right)\right)\right)\right), \\
& \left(\begin{array}{c}
\mathrm{e}^{-1}\left(p \mathrm{e}\left(t-\left(\mathrm{e}^{-1}\left(w_{i} \mathrm{\varrho}\left(t-a_{i}\right)\right)\right)\right)\right. \\
\left.+q \mathrm{e}\left(t-\left(\mathrm{e}^{-1}\left(w_{i} \mathrm{e}\left(t-a_{i}\right)\right)\right)\right)\right)
\end{array}\right) \leq\left(\begin{array}{c}
\mathrm{e}^{-1}\left(p \mathrm{e}\left(t-\left(\mathrm{e}^{-1}\left(w_{i} \mathrm{\varrho}\left(t-\tau_{i}\right)\right)\right)\right)\right. \\
\left.+q \mathrm{e}\left(t-\left(\mathrm{e}^{-1}\left(w_{i} \mathrm{\varrho}\left(t-\tau_{i}\right)\right)\right)\right)\right)
\end{array}\right), \\
& \left(\begin{array}{c}
\mathrm{e}\left(t-\mathrm{e}^{-1}\left(p \mathrm{e}\left(t-\left(\mathrm{e}^{-1}\left(\omega_{i} \mathrm{e}\left(t-a_{i}\right)\right)\right)\right)\right.\right. \\
\left(+q \mathrm{e}\left(t-\left(\mathrm{\varrho}^{-1}\left(\omega_{j} \mathrm{e}\left(t-a_{j}\right)\right)\right)\right)\right)
\end{array}\right) \leq\left(\begin{array}{c}
\mathrm{e}\left(t-\mathrm{e}^{-1}\left(p \mathrm{e}\left(t-\left(\mathrm{e}^{-1}\left(\omega_{i} \mathrm{e}\left(t-\tau_{i}\right)\right)\right)\right)\right.\right. \\
\left.\left.+q \mathrm{e}\left(t-\left(\mathrm{e}^{-1}\left(\omega_{j} \mathrm{e}\left(t-\tau_{j}\right)\right)\right)\right)\right)\right)
\end{array}\right) \\
& \left(\begin{array}{c}
\sum_{i=1}^{n} \sum_{j=1}^{i} \mathrm{e}\left(t-\mathrm{e}^{-1}\left(p \mathrm{e}\left(t-\left(\mathrm{e}^{-1}\left(\omega_{i} \mathrm{e}\left(t-a_{i}\right)\right)\right)\right)\right.\right. \\
\left.\left.+q \mathrm{\varrho}\left(t-\left(\mathrm{e}^{-1}\left(\omega_{j} \mathrm{e}\left(t-a_{j}\right)\right)\right)\right)\right)\right)
\end{array}\right) \leq\left(\begin{array}{c}
\sum_{i=1}^{n} \sum_{j=1}^{i} \mathrm{e}\left(t-\mathrm{e}^{-1}\left(p \mathrm{e}\left(t-\left(\omega_{i} \mathrm{e}\left(t-\tau_{i}\right)\right)\right)\right)\right. \\
\left.\left.+q \mathrm{e}\left(t-\left(\mathrm{e}^{-1}\left(\omega_{j} \mathrm{e}\left(t-\tau_{j}\right)\right)\right)\right)\right)\right)
\end{array}\right), \\
& \left(\begin{array}{c}
\mathrm{e}^{-1}\left(\frac { 2 } { n ( n + 1 ) } \sum _ { i = 1 } ^ { n } \sum _ { j = 1 } ^ { i } \mathrm { \varrho } \left(t-\mathrm{e}^{-1}\left(p \mathrm{e}\left(t-\left(\mathrm{e}^{-1}\left(\omega_{i} \mathrm{e}\left(t-a_{i}\right)\right)\right)\right)\right.\right.\right. \\
\left.\left.\left.+q \mathrm{e}\left(t-\left(\mathrm{e}^{-1}\left(\omega_{j} \mathrm{e}\left(t-a_{j}\right)\right)\right)\right)\right)\right)\right)
\end{array}\right) \geq\left(\begin{array}{c}
\mathrm{e}^{-1}\left(\frac { 2 } { n ( n + 1 ) } \sum _ { i = 1 } ^ { n } \sum _ { j = 1 } ^ { i } \mathrm { e } \left(t-\mathrm{e}^{-1}\left(p \mathrm{e}\left(t-\left(\mathrm{e}^{-1}\left(\omega_{i} \mathrm{e}\left(t-\tau_{i}\right)\right)\right)\right)\right.\right.\right. \\
\left.\left.\left.+q \mathrm{e}\left(t-\left(\mathrm{e}^{-1}\left(\omega_{j} \mathrm{e}\left(t-\tau_{j}\right)\right)\right)\right)\right)\right)\right)
\end{array}\right),
\end{aligned}
$$

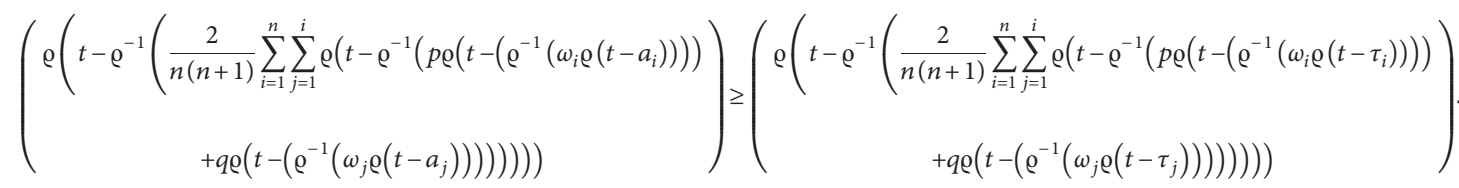

$$
\begin{aligned}
& \left(\begin{array}{c}
\mathrm{e}^{-1}\left(\frac { 1 } { ( p + q ) } \mathrm { e } \left(t-\mathrm{e}^{-1}\left(\frac { 2 } { n ( n + 1 ) } \sum _ { i = 1 } ^ { n } \sum _ { j = 1 } ^ { i } \mathrm { \varrho } \left(t-\mathrm{e}^{-1}\left(p \mathrm{e}\left(t-\left(\mathrm{e}^{-1}\left(\omega_{i} \mathrm{e}\left(t-a_{i}\right)\right)\right)\right)\right.\right.\right.\right.\right. \\
\left.\left.\left.\left.\left.+q \mathrm{e}\left(t-\left(\mathrm{\varrho}^{-1}\left(\omega_{j} \mathrm{e}\left(t-a_{j}\right)\right)\right)\right)\right)\right)\right)\right)\right)
\end{array}\right) \leq\left(\begin{array}{c}
\mathrm{e}^{-1}\left(\frac { 1 } { ( p + q ) } \mathrm { e } \left(t-\mathrm{e}^{-1}\left(\frac { 2 } { n ( n + 1 ) } \sum _ { i = 1 } ^ { n } \sum _ { j = 1 } ^ { i } \mathrm { e } \left(t-\mathrm{e}^{-1}\left(p \mathrm{e}\left(t-\left(\mathrm{e}^{-1}\left(\omega_{i} \mathrm{e}\left(t-\tau_{i}\right)\right)\right)\right)\right.\right.\right.\right.\right. \\
\left.\left.\left.\left.\left.+q \mathrm{e}\left(t-\left(\mathrm{e}^{-1}\left(\omega_{j} \mathrm{e}\left(t-\tau_{j}\right)\right)\right)\right)\right)\right)\right)\right)\right)
\end{array}\right) .
\end{aligned}
$$

Similarly, we have

$$
\begin{aligned}
& \left(\begin{array}{c}
\varrho^{-1}\left(\frac { 1 } { ( p + q ) } \varrho \left(t-\varrho^{-1}\left(\frac { 2 } { n ( n + 1 ) } \sum _ { i = 1 } ^ { n } \sum _ { j = 1 } ^ { i } \varrho \left(t-\varrho^{-1}\left(p \varrho\left(t-\left(\varrho^{-1}\left(\omega_{i} \varrho\left(t-b_{i}\right)\right)\right)\right)\right.\right.\right.\right.\right. \\
\left.\left.\left.\left.\left.+q \varrho\left(t-\left(\varrho^{-1}\left(\omega_{j} \varrho\left(t-b_{j}\right)\right)\right)\right)\right)\right)\right)\right)\right)
\end{array}\right) \\
& \leq\left(\begin{array}{c}
\varrho^{-1}\left(\frac { 1 } { ( p + q ) } \varrho \left(t-\varrho^{-1}\left(\frac { 2 } { n ( n + 1 ) } \sum _ { i = 1 } ^ { n } \sum _ { j = 1 } ^ { i } \varrho \left(t-\varrho^{-1}\left(p \varrho\left(t-\left(\varrho^{-1}\left(\omega_{i} \varrho\left(t-\theta_{i}\right)\right)\right)\right)\right.\right.\right.\right.\right. \\
\left.\left.\left.\left.\left.+q \varrho\left(t-\left(\varrho^{-1}\left(\omega_{j} \varrho\left(t-\theta_{j}\right)\right)\right)\right)\right)\right)\right)\right)\right)
\end{array}\right)
\end{aligned}
$$


On the other hand, as $c_{i} \geq \eta_{i}$ and $d_{i} \geq v_{i}$, we have $\varrho\left(c_{i}\right) \leq \varrho\left(\eta_{i}\right), \varrho^{-1}\left(w_{i} \varrho\left(c_{i}\right)\right) \geq \varrho^{-1}\left(w_{i} \varrho\left(\eta_{i}\right)\right)$, and $p \varrho\left(t-\varrho^{-1}\right.$ $\left(\omega_{i} \varrho\left(c_{i}\right)\right) \geq p \varrho\left(t-\varrho^{-1}\left(\omega_{i} \varrho\left(\eta_{i}\right)\right)\right.$.

$$
\begin{aligned}
& \left(\begin{array}{c}
\mathrm{\varrho}^{-1}\left(p \varrho\left(t-\varrho^{-1}\left(\omega_{i} \varrho\left(c_{i}\right)\right)\right)\right. \\
\left.+q \varrho\left(t-\varrho^{-1}\left(\omega_{j} \varrho\left(c_{j}\right)\right)\right)\right)
\end{array}\right) \leq\left(\begin{array}{c}
\varrho^{-1}\left(p \varrho\left(t-\varrho^{-1}\left(\omega_{i} \varrho\left(\eta_{i}\right)\right)\right)\right. \\
\left.+q \varrho\left(t-\varrho^{-1}\left(\omega_{j} \varrho\left(\eta_{j}\right)\right)\right)\right)
\end{array}\right), \\
& \left(\begin{array}{c}
\sum_{i=1}^{n} \sum_{j=1}^{i} \varrho\left(t-\varrho^{-1}\left(p \varrho\left(t-\varrho^{-1}\left(\omega_{i} \varrho\left(c_{i}\right)\right)\right)\right.\right. \\
\left.\left.+q \varrho\left(t-\varrho^{-1}\left(\omega_{j} \varrho\left(c_{j}\right)\right)\right)\right)\right)
\end{array}\right) \leq\left(\begin{array}{c}
\sum_{i=1}^{n} \sum_{j=1}^{i} \varrho\left(t-\varrho^{-1}\left(p \varrho\left(t-\varrho^{-1}\left(\omega_{i} \varrho\left(\eta_{i}\right)\right)\right)\right.\right. \\
\left.\left.+q \varrho\left(t-\varrho^{-1}\left(\omega_{j} \varrho\left(\eta_{j}\right)\right)\right)\right)\right)
\end{array}\right) \\
& \left(\begin{array}{c}
\varrho\left(t-\varrho^{-1}\left(\frac { 2 } { n ( n + 1 ) } \sum _ { i = 1 } ^ { n } \sum _ { j = 1 } ^ { i } \varrho \left(t-\varrho^{-1}\left(p \varrho\left(t-\varrho^{-1}\left(\omega_{i} \varrho\left(c_{i}\right)\right)\right)\right.\right.\right.\right. \\
\left.\left.\left.\left.+q \varrho\left(t-\varrho^{-1}\left(\omega_{j} \varrho\left(c_{j}\right)\right)\right)\right)\right)\right)\right)
\end{array}\right) \geq\left(\begin{array}{c}
\varrho\left(t-\varrho^{-1}\left(\frac { 2 } { n ( n + 1 ) } \sum _ { i = 1 } ^ { n } \sum _ { j = 1 } ^ { i } \varrho \left(t-\varrho^{-1}\left(p \varrho\left(t-\varrho^{-1}\left(\omega_{i} \varrho\left(\eta_{i}\right)\right)\right)\right.\right.\right.\right. \\
\left.\left.\left.\left.+q \varrho\left(t-\varrho^{-1}\left(\omega_{j} \varrho\left(\eta_{j}\right)\right)\right)\right)\right)\right)\right)
\end{array}\right), \\
& \left.\begin{array}{c}
t-\left(\varrho ^ { - 1 } \left(\frac { 1 } { ( p + q ) } \varrho \left(t-\varrho^{-1}\left(\frac { 2 } { n ( n + 1 ) } \sum _ { i = 1 } ^ { n } \sum _ { j = 1 } ^ { i } \varrho \left(t-\varrho^{-1}\left(p \varrho\left(t-\varrho^{-1}\left(\omega_{i} \varrho\left(c_{i}\right)\right)\right)\right.\right.\right.\right.\right.\right. \\
\left.\left.\left.\left.\left.\left.+q \varrho\left(t-\varrho^{-1}\left(\omega_{j} \varrho\left(c_{j}\right)\right)\right)\right)\right)\right)\right)\right)\right)
\end{array}\right) \geq\left(\begin{array}{c}
t-\left(\varrho ^ { - 1 } \left(\frac { 1 } { ( p + q ) } \varrho \left(t-\varrho^{-1}\left(\frac { 2 } { n ( n + 1 ) } \sum _ { i = 1 } ^ { n } \sum _ { j = 1 } ^ { i } \varrho \left(t-\varrho^{-1}\left(p \varrho\left(t-\varrho^{-1}\left(\omega_{i} \varrho\left(\eta_{i}\right)\right)\right)\right)\right.\right.\right.\right.\right. \\
\left.\left.\left.\left.\left.\left.+q \varrho\left(t-\varrho^{-1}\left(\omega_{j} \varrho\left(\eta_{j}\right)\right)\right)\right)\right)\right)\right)\right)\right)
\end{array}\right)
\end{aligned}
$$

Similarly, we have

$$
\left.\begin{array}{c}
\left(t-\left(\varrho ^ { - 1 } \left(\frac { 1 } { ( p + q ) } \varrho \left(t-\varrho^{-1}\left(\frac { 2 } { n ( n + 1 ) } \sum _ { i = 1 } ^ { n } \sum _ { j = 1 } ^ { i } \varrho \left(t-\varrho^{-1}\left(p \varrho\left(t-\varrho^{-1}\left(\omega_{i} \varrho\left(d_{i}\right)\right)\right)\right)\right.\right.\right.\right.\right.\right. \\
\left.\left.\left.\left.\left.\left.+q \varrho\left(t-\varrho^{-1}\left(\omega_{j} \varrho\left(d_{j}\right)\right)\right)\right)\right)\right)\right)\right)\right)
\end{array}\right) .
$$

Therefore, LIVIFWCHM ${ }^{p, q}\left(\alpha_{1}, \ldots, \alpha_{n}\right) \leq \operatorname{LIVIFWCHM}^{p, q}$ $\left(\beta_{1}, \ldots, \beta_{n}\right)$.
Theorem 6 (boundness). Let $\alpha_{i}=\left(\left[s_{a_{i}}, s_{b_{i}}\right],\left[s_{c_{i}}, s_{d_{i}}\right]\right)$ be a collection of LIVIFNs; if $\alpha^{+},\left(\left[\max \left(s_{a_{i}}\right), \max \left(s_{b_{i}}\right)\right]\right.$, $\left.\left[\min \left(s_{c_{i}}\right), \min \left(s_{d_{i}}\right)\right]\right) \quad$ and $\alpha^{-},\left(\left[\min \left(s_{a_{i}}\right), \min \left(s_{b_{i}}\right)\right]\right.$, $\left.\left[\max \left(s_{c_{i}}\right), \max \left(s_{d_{i}}\right)\right]\right)$ for all $i$, then

$$
\operatorname{LIVIFWCHM}^{p, q}\left(\alpha^{-}, \ldots, \alpha^{-}\right) \leq \operatorname{LIVIFWCHM}^{p, q}\left(\beta_{1}, \ldots, \beta_{n}\right) \leq \operatorname{LIVIFWCHM}^{p, q}\left(\alpha^{+}, \ldots, \alpha^{+}\right) .
$$

Proof. According to Theorem 5, the conclusion is obvious, so we omitted it here.

According to formula (20) and Theorem 4, it is easy to know that operators do not satisfy idempotency.
Now, we can discuss some special cases of the LIVIFWCHM ${ }^{p, q}$ operator with respect to the parameters $p$ and $q$.

(1) When $q \longrightarrow 0$, the formula reduces to 
$\operatorname{LIVIFWCHM}^{p, q}\left(\alpha_{1}, \ldots, \alpha_{n}\right)=\left(\left[s_{a}, s_{b}\right],\left[s_{c}, s_{d}\right]\right)$,

$$
\begin{aligned}
& a=\varrho^{-1}\left(\frac{1}{p} \varrho\left(t-\varrho^{-1}\left(\frac{2}{n(n+1)} \sum_{i=1}^{n} \varrho^{-1}\left((n+1-i) \varrho\left(t-\varrho^{-1}\left(p \varrho\left(t-\left(\varrho^{-1}\left(\omega_{i} \varrho\left(t-a_{i}\right)\right)\right)\right)\right)\right)\right)\right)\right)\right), \\
& a=\varrho^{-1}\left(\frac{1}{p} \varrho\left(t-\varrho^{-1}\left(\frac{2}{n(n+1)} \sum_{i=1}^{n} \varrho^{-1}\left((n+1-i) \varrho\left(t-\varrho^{-1}\left(p \varrho\left(t-\left(\varrho^{-1}\left(\omega_{i} \varrho\left(t-b_{i}\right)\right)\right)\right)\right)\right)\right)\right)\right)\right), \\
& c=t-\left(\varrho^{-1}\left(\frac{1}{p} \varrho\left(t-\varrho^{-1}\left(\frac{2}{n(n+1)} \sum_{i=1}^{n} \varrho^{-1}\left((n+1-i) \varrho\left(t-\varrho^{-1}\left(p \varrho\left(t-\varrho^{-1}\left(\omega_{i} \varrho\left(c_{i}\right)\right)\right)\right)\right)\right)\right)\right)\right)\right), \\
& c=t-\left(\varrho^{-1}\left(\frac{1}{p} \varrho\left(t-\varrho^{-1}\left(\frac{2}{n(n+1)} \sum_{i=1}^{n} \varrho^{-1}\left((n+1-i) \varrho\left(t-\varrho^{-1}\left(p \varrho\left(t-\varrho^{-1}\left(\omega_{i} \varrho\left(d_{i}\right)\right)\right)\right)\right)\right)\right)\right)\right)\right) .
\end{aligned}
$$

(2) When $p \longrightarrow 0$, the formula reduces to

$\operatorname{LIVIFWCHM}^{p, q}\left(\alpha_{1}, \ldots, \alpha_{n}\right)=\left(\left[s_{a}, s_{b}\right],\left[s_{c}, s_{d}\right]\right)$,

$$
\begin{aligned}
& a=\varrho^{-1}\left(\frac{1}{q} \varrho\left(t-\varrho^{-1}\left(\frac{2}{n(n+1)} \sum_{i=1}^{n} \varrho^{-1}\left(i \varrho\left(t-\varrho^{-1}\left(q \varrho\left(t-\left(\varrho^{-1}\left(\omega_{i} \varrho\left(t-a_{i}\right)\right)\right)\right)\right)\right)\right)\right)\right)\right) \\
& a=\varrho^{-1}\left(\frac{1}{q} \varrho\left(t-\varrho^{-1}\left(\frac{2}{n(n+1)} \sum_{i=1}^{n} \varrho^{-1}\left(i \varrho\left(t-\varrho^{-1}\left(q \varrho\left(t-\left(\varrho^{-1}\left(\omega_{i} \varrho\left(t-b_{i}\right)\right)\right)\right)\right)\right)\right)\right)\right)\right) \\
& c=t-\left(\varrho^{-1}\left(\frac{1}{q} \varrho\left(t-\varrho^{-1}\left(\frac{2}{n(n+1)} \sum_{i=1}^{n} \varrho^{-1}\left(i \varrho\left(t-\varrho^{-1}\left(q \varrho\left(t-\varrho^{-1}\left(\omega_{i} \varrho\left(c_{i}\right)\right)\right)\right)\right)\right)\right)\right)\right)\right) \\
& c=t-\left(\varrho^{-1}\left(\frac{1}{q} \varrho\left(t-\varrho^{-1}\left(\frac{2}{n(n+1)} \sum_{i=1}^{n} \varrho^{-1}\left(i \varrho\left(t-\varrho^{-1}\left(q \varrho\left(t-\varrho^{-1}\left(\omega_{i} \varrho\left(d_{i}\right)\right)\right)\right)\right)\right)\right)\right)\right)\right) .
\end{aligned}
$$

(3) When $p=q=(1 / 2)$, the formula reduces to an interval-valued intuitionistic fuzzy basic Heronian operator.

(4) When $p=q=1$, the formula reduces to an intervalvalued intuitionistic fuzzy basic line Heronian mean operator.
Some different types of LIVIFWCHM ${ }^{p, q}$ are as follows: Case 1: Gumbel type: when $\varrho(c)=(-\ln (c / t))^{\theta}$, $\mathrm{Q}^{-1}(c)=\mathrm{te}^{-c(1 / \theta)}$, and $\theta \geq 1$, we have

$$
G-\operatorname{LIVIFWCHM}^{p, q}\left(\alpha_{1}, \ldots, \alpha_{n}\right)=\left(\left[s_{a}, s_{b}\right],\left[s_{c}, s_{d}\right]\right) \text {, }
$$


where

$$
\begin{aligned}
& a=\mathrm{te}-\left(\frac{1}{p+q}\left(-\ln \left(1-e^{-a_{4}^{(1 / \theta)}}\right)\right)^{\theta}\right)^{(1 / \theta)}, \\
& b=\mathrm{te}-\left(\frac{1}{p+q}\left(-\ln \left(1-e^{-b_{4}^{(1 / \theta)}}\right)\right)^{\theta}\right)^{(1 / \theta)}, \\
& c=t-\text { te }-\left(\frac{1}{p+q}\left(-\ln \left(1-e^{-c_{4}^{(1 / \theta)}}\right)\right)^{\theta}\right)^{(1 / \theta)}, \\
& c=t-\mathrm{te}-\left(\frac{1}{p+q}\left(-\ln \left(1-e^{-d_{4}^{(1 / \theta)}}\right)\right)^{\theta}\right)^{(1 / \theta)} \\
& a_{4}=\frac{2}{n(n+1)} \sum_{i=1}^{n} \sum_{j=1}^{i}\left(-\ln \left(1-e^{-a_{4}^{\prime(1 / \theta)}}\right)\right)^{\theta} \\
& b_{4}=\frac{2}{n(n+1)} \sum_{i=1}^{n} \sum_{j=1}^{i}\left(-\ln \left(1-e^{-b_{4}^{(1 / \theta)}}\right)\right)^{\theta} \text {, } \\
& c_{4}=\frac{2}{n(n+1)} \sum_{i=1}^{n} \sum_{j=1}^{i}\left(-\ln \left(1-e^{-c_{4}^{(1 / \theta)}}\right)\right)^{\theta} \\
& d_{4}=\frac{2}{n(n+1)} \sum_{i=1}^{n} \sum_{j=1}^{i}\left(-\ln \left(1-e^{-d_{4}^{(1 / \theta)}}\right)\right)^{\theta} \\
& a_{4}^{\prime}=p\left(-\ln \left(1-e^{-\left(w_{i}\left(-\ln \left(t-a_{i} / t\right)\right)^{\theta}\right)^{(1 / \theta)}}\right)\right)^{\theta}+q\left(-\ln \left(1-e^{-\left(w_{j}\left(-\ln \left(t-a_{j} / t\right)\right)^{\theta}\right)^{(1 / \theta)}}\right)\right)^{\theta} \\
& b_{4}^{\prime}=p\left(-\ln \left(1-e^{-\left(w_{i}\left(-\ln \left(t-b_{i} / t\right)\right)^{\theta}\right)^{(1 / \theta)}}\right)\right)^{\theta}+q\left(-\ln \left(1-e^{-\left(w_{j}\left(-\ln \left(t-b_{j} / t\right)\right)^{\theta}\right)^{(1 / \theta)}}\right)\right)^{\theta} \\
& c_{4}^{\prime}=p\left(-\ln \left(1-e^{-\left(w_{i}\left(-\ln \left(c_{i} / t\right)\right)^{\theta}\right)^{(1 / \theta)}}\right)\right)^{\theta}+q\left(-\ln \left(1-e^{-\left(w_{j}\left(-\ln \left(c_{j} / t\right)\right)^{\theta}\right)^{(1 / \theta)}}\right)\right)^{\theta}, \\
& d_{4}^{\prime}=p\left(-\ln \left(1-e^{-\left(w_{i}\left(-\ln \left(d_{i} / t\right)\right)^{\theta}\right)^{(1 / \theta)}}\right)\right)^{\theta}+q\left(-\ln \left(1-e^{-\left(w_{j}\left(-\ln \left(d_{j} / t\right)\right)^{\theta}\right)^{(1 / \theta)}}\right)\right)^{\theta} .
\end{aligned}
$$

Case 2: Clayton type: when $\varrho(c)=(c / t)^{-\theta}-1$, where $\varrho^{-1}(c)=t(c+1)^{-(1 / \theta)}, \theta \geq-1$, and $\theta \neq 0$, we have
$C-\operatorname{LIVIFWCHM}^{p, q}\left(\alpha_{1}, \alpha_{2}, \ldots, \alpha_{n}\right)=\left(\left[s_{a}, s_{b}\right],\left[s_{c}, s_{d}\right]\right)$, 
where

$$
\begin{aligned}
& a=t\left(\frac{1}{p+q}\left(\left(\frac{t-t\left(a_{4}+1\right)^{-(1 / \theta)}}{t}\right)^{-\theta}-1\right)+1\right)^{-(1 / \theta)}, \\
& b=t\left(\frac{1}{p+q}\left(\left(\frac{t-t\left(b_{4}+1\right)^{-(1 / \theta)}}{t}\right)^{-\theta}-1\right)+1\right)^{-(1 / \theta)}, \\
& c=t-t\left(\frac{1}{p+q}\left(\left(\frac{t-t\left(c_{4}+1\right)^{-(1 / \theta)}}{t}\right)^{-\theta}-1\right)+1\right)^{-(1 / \theta)}, \\
& d=t-t\left(\frac{1}{p+q}\left(\left(\frac{t-t\left(d_{4}+1\right)^{-(1 / \theta)}}{t}\right)^{-\theta}-1\right)+1\right)^{-(1 / \theta)}, \\
& a_{4}=\frac{2}{n(n+1)} \sum_{i=1}^{n} \sum_{j=1}^{i}\left(\frac{t-t\left(a_{4}^{\prime}+1\right)^{-(1 / \theta)}}{t}\right)^{-\theta}-1 \text {, } \\
& b_{4}=\frac{2}{n(n+1)} \sum_{i=1}^{n} \sum_{j=1}^{i}\left(\frac{t-t\left(b_{4}^{\prime}+1\right)^{-(1 / \theta)}}{t}\right)^{-\theta}-1, \\
& c_{4}=\frac{2}{n(n+1)} \sum_{i=1}^{n} \sum_{j=1}^{i}\left(\frac{t-t\left(c_{4}^{\prime}+1\right)^{-(1 / \theta)}}{t}\right)^{-\theta}-1, \\
& d_{4}=\frac{2}{n(n+1)} \sum_{i=1}^{n} \sum_{j=1}^{i}\left(\frac{t-t\left(d_{4}^{\prime}+1\right)^{-(1 / \theta)}}{t}\right)^{-\theta}-1, \\
& a_{4}^{\prime}=p\left(\frac{t-t\left(w_{i}\left(\left(t-a_{i} / t\right)^{-\theta}-1\right)+1\right)^{-(1 / \theta)}}{t}\right)^{-\theta}+q\left(\frac{t-t\left(w_{j}\left(\left(t-a_{j} / t\right)^{-\theta}-1\right)+1\right)^{-(1 / \theta)}}{t}\right)^{-\theta}-2 \\
& b_{4}^{\prime}=p\left(\frac{t-t\left(w_{i}\left(\left(t-b_{i} / t\right)^{-\theta}-1\right)+1\right)^{-(1 / \theta)}}{t}\right)^{-\theta}+q\left(\frac{t-t\left(w_{j}\left(\left(t-b_{j} / t\right)^{-\theta}-1\right)+1\right)^{-(1 / \theta)}}{t}\right)^{-\theta}-2 \\
& c_{4}^{\prime}=p\left(\frac{t-t\left(w_{i}\left(\left(c_{i} / t\right)^{-\theta}-1\right)+1\right)^{-(1 / \theta)}}{t}\right)^{-\theta}+q\left(\frac{t-t\left(w_{j}\left(\left(c_{j} / t\right)^{-\theta}-1\right)+1\right)^{-(1 / \theta)}}{t}\right)^{-\theta}-2, \\
& d_{4}^{\prime}=p\left(\frac{t-t\left(w_{i}\left(\left(d_{i} / t\right)^{-\theta}-1\right)+1\right)^{-(1 / \theta)}}{t}\right)^{-\theta}+q\left(\frac{t-t\left(w_{j}\left(\left(d_{j} / t\right)^{-\theta}-1\right)+1\right)^{-(1 / \theta)}}{t}\right)^{-\theta}-2 .
\end{aligned}
$$


Case 3: Frank type: when $\varrho(c)=\ln \left(e^{-(\theta c / t)}-1 /\right.$ where $\left.e^{-\theta}-1\right), \quad \varrho^{-1}(c)=(-(t / \theta)) \ln \left(e^{c}\left(e^{-\theta}-1\right)+1\right), \quad$ and $\theta \neq 0$, we have

$F-\operatorname{LIVIFWCHM}^{p, q}\left(\alpha_{1}, \alpha_{2}, \ldots, \alpha_{n}\right)=\left(\left[s_{a}, s_{b}\right],\left[s_{c}, s_{d}\right]\right)$,

$$
\begin{aligned}
& a=-\frac{t}{\theta} \ln \left(\left(\left(\frac{e^{-\left(\theta a_{4} / t\right)}-1}{e^{-\theta}-1}\right)\right)^{(1 / p+q)}\left(e^{-\theta}-1\right)+1\right), b \\
& c=t+\frac{t}{\theta} \ln \left(\left(\left(\frac{e^{-\left(\theta a_{4} / t\right)}-1}{e^{-\theta}-1}\right)\right)^{(1 / p+q)}\left(e^{-\theta}-1\right)+1\right), d \\
& a_{4}=t+\frac{t}{\theta} \ln \left(\left(\prod_{i=1}^{n} \prod_{j=1}^{i}\left(\frac{e^{-\left(\theta_{4 i j}^{\prime} / t\right)}-1}{e^{-\theta}-1}\right)\right)^{(2 / n(n+1))}\left(e^{-\theta}-1\right)+1\right) \text {, } \\
& b_{4}=t+\frac{t}{\theta} \ln \left(\left(\prod_{i=1}^{n} \prod_{j=1}^{i}\left(\frac{e^{-\left(\theta b_{4 i} / t\right)}-1}{e^{-\theta}-1}\right)\right)^{(2 / n(n+1))}\left(e^{-\theta}-1\right)+1\right) \text {, } \\
& c_{4}=t+\frac{t}{\theta} \ln \left(\left(\prod_{i=1}^{n} \prod_{j=1}^{i}\left(\frac{e^{-\left(\theta_{c_{4 i}}^{\prime} / t\right)}-1}{e^{-\theta}-1}\right)\right)^{(2 / n(n+1))}\left(e^{-\theta}-1\right)+1\right) \text {, } \\
& d_{4}=t+\frac{t}{\theta} \ln \left(\left(\prod_{i=1}^{n} \prod_{j=1}^{i}\left(\frac{e^{-\left(\theta d_{4 i} / t\right)}-1}{e^{-\theta}-1}\right)\right)^{(2 / n(n+1))}\left(e^{-\theta}-1\right)+1\right) \text {, } \\
& a_{4 i j}^{\prime}=t+\frac{t}{\theta} \ln \left(\left(\frac{e^{-\left(\theta a_{4}^{\prime} / t\right)}-1}{e^{-\theta}-1}\right)^{p}\left(\frac{e^{-\left(\theta \dot{a}_{4} / t\right)}-1}{e^{-\theta}-1}\right)^{q}\left(e^{-\theta}-1\right)+1\right) \text {, } \\
& b_{4 i j}^{\prime}=t+\frac{t}{\theta} \ln \left(\left(\frac{e^{-\left(\theta b_{4} / t\right)}-1}{e^{-\theta}-1}\right)^{p}\left(\frac{e^{-\left(\theta b_{4 j}^{\prime} / t\right)}-1}{e^{-\theta}-1}\right)^{q}\left(e^{-\theta}-1\right)+1\right) \text {, } \\
& a_{4 i}^{\prime}=t+\frac{t}{\theta} \ln \left(\left(\frac{e^{-\left(\theta\left(t-a_{i}\right) / t\right)}-1}{e^{-\theta}-1}\right)^{w_{i}}\left(e^{-\theta}-1\right)+1\right), a_{4 j}^{\prime}=t+\frac{t}{\theta} \ln \left(\left(\frac{e^{-\left(\theta\left(t-a_{j}\right) / t\right.}-1}{e^{-\theta}-1}\right)^{w_{j}}\left(e^{-\theta}-1\right)+1\right) \text {, } \\
& b_{4 i}^{\prime}=t+\frac{t}{\theta} \ln \left(\left(\frac{e^{-\left(\theta\left(t-b_{i}\right) / t\right)}-1}{e^{-\theta}-1}\right)^{w_{i}}\left(e^{-\theta}-1\right)+1\right), b_{4 j}^{\prime}=t+\frac{t}{\theta} \ln \left(\left(\frac{e^{-\left(\theta\left(t-b_{j}\right) / t\right.}-1}{e^{-\theta}-1}\right)^{w_{j}}\left(e^{-\theta}-1\right)+1\right) \text {, } \\
& c_{4 i j}^{\prime}=t+\frac{t}{\theta} \ln \left(\left(\frac{e^{-\left(\theta c_{4}^{\prime} / t\right)}-1}{e^{-\theta}-1}\right)^{p}\left(\frac{e^{-\left(\theta c_{4 j}^{\prime} / t\right)}-1}{e^{-\theta}-1}\right)^{q}\left(e^{-\theta}-1\right)+1\right) \\
& d_{4 i j}^{\prime}=t+\frac{t}{\theta} \ln \left(\left(\frac{e^{-\left(\theta \dot{d}_{4 j} / t\right)}-1}{e^{-\theta}-1}\right)^{p}\left(\frac{e^{-\left(\theta d_{4 j}^{\prime} / t\right)}-1}{e^{-\theta}-1}\right)^{q}\left(e^{-\theta}-1\right)+1\right) \text {, } \\
& c_{4 i}^{\prime}=t+\frac{t}{\theta} \ln \left(\left(\frac{e^{-\left(\theta\left(c_{i}\right) / t\right)}-1}{e^{-\theta}-1}\right)^{w_{i}}\left(e^{-\theta}-1\right)+1\right), c_{4 j}^{\prime}=t+\frac{t}{\theta} \ln \left(\left(\frac{e^{-\left(\theta\left(c_{j}\right) / t\right)}-1}{e^{-\theta}-1}\right)^{w_{j}}\left(e^{-\theta}-1\right)+1\right), \\
& d_{4 i}^{\prime}=t+\frac{t}{\theta} \ln \left(\left(\frac{e^{-\left(\theta\left(d_{i}\right) / t\right)}-1}{e^{-\theta}-1}\right)^{w_{i}}\left(e^{-\theta}-1\right)+1\right), d_{4 j}^{\prime}=t+\frac{t}{\theta} \ln \left(\left(\frac{e^{--\left(\theta\left(d_{j}\right) / t\right)}-1}{e^{-\theta}-1}\right)^{w_{j}}\left(e^{-\theta}-1\right)+1\right) \text {. }
\end{aligned}
$$


Case 4: Ali-Mikhail-Haq type: when $\varrho(c)=\ln (t-\theta(t-c) /$

where

$c), \varrho^{-1}(c)=\left(t(1-\theta) / e^{c}-\theta\right)$, and $\theta \in[-1,1)$, we have

$A-\operatorname{LIVIFWCHM}^{p, q}\left(\alpha_{1}, \alpha_{2}, \ldots, \alpha_{n}\right)=\left(\left[s_{a}, s_{b}\right],\left[s_{c}, s_{d}\right]\right)$,

$$
\begin{aligned}
& a=\frac{t(1-\theta)}{\left(t-\theta a_{4} / t-a_{4}\right)^{(1 / p+q)}-\theta}, b \\
& c=t-\frac{t(1-\theta)}{\left(t-\theta c_{4} / t-c_{4}\right)^{(1 / p+q)}-\theta}, d \\
& a_{4}=\frac{t(1-\theta)}{\left(\prod_{i=1}^{n} \prod_{j=1}^{i}\left(t-\theta a_{4 i j}^{\prime} / t-a_{4 i j}^{\prime}\right)\right)^{(2 / n(n+1))}-\theta}, \\
& b_{4}=\frac{t(1-\theta)}{\left(\prod_{i=1}^{n} \prod_{j=1}^{i}\left(t-\theta b_{4 i j}^{\prime} / t-b_{4 i j}^{\prime}\right)\right)^{(2 / n(n+1))}-\theta}, \\
& c_{4}=\frac{t(1-\theta)}{\left(\prod_{i=1}^{n} \prod_{j=1}^{i}\left(t-\theta c_{4 i j}^{\prime} / t-a_{4 i j}^{\prime}\right)\right)^{(2 / n(n+1))}-\theta}, \\
& d_{4}=\frac{t(1-\theta)}{\left(\prod_{i=1}^{n} \prod_{j=1}^{i}\left(t-\theta d_{4 i j}^{\prime} / t-d_{4 i j}^{\prime}\right)\right)^{(2 / n(n+1))}-\theta}, \\
& a_{4 i j}^{\prime}=\frac{t(1-\theta)}{\left(t-\theta a_{4 i}^{\prime} / t-a_{4 i}^{\prime}\right)^{p}\left(t-\theta a_{4 j}^{\prime} / t-a_{4 j}^{\prime}\right)^{q}-\theta}, \\
& b_{4 i j}^{\prime}=\frac{t(1-\theta)}{\left(t-\theta b_{4 i}^{\prime} / t-a_{4 i}^{\prime}\right)^{p}\left(t-\theta b_{4 j}^{\prime} / t-a_{4 j}^{\prime}\right)^{q}-\theta}, \\
& c_{4 i j}^{\prime}=\frac{t(1-\theta)}{\left(t-\theta c_{4 i}^{\prime} / t-c_{4 i}^{\prime}\right)^{p}\left(t-\theta c_{4 j}^{\prime} / t-c_{4 j}^{\prime}\right)^{q}-\theta}, \\
& d_{4 i j}^{\prime}=\frac{t(1-\theta)}{\left(t-\theta d_{4 i}^{\prime} / t-c_{4 i}^{\prime}\right)^{p}\left(t-\theta d_{4 j}^{\prime} / t-d_{4 j}^{\prime}\right)^{q}-\theta}, \\
& a_{4 i}^{\prime}=\frac{t(1-\theta)}{\left(t-\theta a_{i} / t-a_{i}\right)^{w_{i}}-\theta}, \\
& a_{4 j}^{\prime}=\frac{t(1-\theta)}{\left(t-\theta a_{j} / t-a_{j}\right)^{w_{j}}-\theta}, \\
& b_{4 i}^{\prime}=\frac{t(1-\theta)}{\left(t-\theta b_{i} / t-b_{i}\right)^{w_{i}}-\theta}, \\
& b_{4 j}^{\prime}=\frac{t(1-\theta)}{\left(t-\theta b_{j} / t-b_{j}\right)^{w_{j}}-\theta}, \\
& c_{4 i}^{\prime}=\frac{t(1-\theta)}{\left(t-\theta\left(t-c_{i}\right) / t-c_{i}\right)^{w_{i}}-\theta}, \\
& c_{4 j}^{\prime}=\frac{t(1-\theta)}{\left(t-\theta\left(t-c_{j}\right) / t-c_{j}\right)^{w_{j}}-\theta}, \\
& d_{4 i}^{\prime}=\frac{t(1-\theta)}{\left(t-\theta\left(t-d_{i}\right) / t-d_{i}\right)^{w_{i}}-\theta}, \\
& d_{4 j}^{\prime}=\frac{t(1-\theta)}{\left(t-\theta\left(t-d_{j}\right) / t-d_{j}\right)^{w_{j}}-\theta} \text {. }
\end{aligned}
$$


Case 5: Joe type: when $\varrho(c)=-\ln \left(1-(1-(c / t))^{\theta}\right)$ and where $\varrho^{-1}(c)=t-t\left(1-e^{-c}\right)^{(1 / \theta)}$, where $\theta \geq 1$, we have $J-\operatorname{LIVIFWCHM}^{p, q}\left(\alpha_{1}, \alpha_{2}, \ldots, \alpha_{n}\right)=\left(\left[s_{a}, s_{b}\right],\left[s_{c}, s_{d}\right]\right)$,

$$
\begin{aligned}
& a=t-t\left(1-\left(1-\left(1-\left(1-a_{4}\right)^{(1 / \theta)}\right)^{\theta}\right)^{(1 / p+q)}\right)^{(1 / \theta)}, \\
& b=t-t\left(1-\left(1-\left(1-\left(1-b_{4}\right)^{(1 / \theta)}\right)^{\theta}\right)^{(1 / p+q)}\right)^{(1 / \theta)}, \\
& c=t\left(1-\left(1-\left(1-\left(1-c_{4}\right)^{(1 / \theta)}\right)^{\theta}\right)^{(1 / p+q)}\right)^{(1 / \theta)}, \\
& d=t\left(1-\left(1-\left(1-\left(1-d_{4}\right)^{(1 / \theta)}\right)^{\theta}\right)^{(1 / p+q)}\right)^{(1 / \theta)}, \\
& a_{4}=\left(\prod_{i=1}^{n} \prod_{j=1}^{i}\left(1-\left(1-\left(1-a_{4}^{\prime}\right)^{(1 / \theta)}\right)^{\theta}\right)\right)^{(2 / n(n+1))}, \\
& b_{4}=\left(\prod_{i=1}^{n} \prod_{j=1}^{i}\left(1-\left(1-\left(1-b_{4}^{\prime}\right)^{(1 / \theta)}\right)^{\theta}\right)\right)^{(2 / n(n+1))}, \\
& c_{4}=\left(\prod_{i=1}^{n} \prod_{j=1}^{i}\left(1-\left(1-\left(1-c_{4}^{\prime}\right)^{(1 / \theta)}\right)^{\theta}\right)\right)^{(2 / n(n+1))}, \\
& d_{4}=\left(\prod_{i=1}^{n} \prod_{j=1}^{i}\left(1-\left(1-\left(1-d_{4}^{\prime}\right)^{(1 / \theta)}\right)^{\theta}\right)\right)^{(2 / n(n+1))}, \\
& d_{4}^{\prime}=\left(1-\left(1-\left(1-\left(1-\left(1-\left(d_{i} / t\right)\right)^{\theta}\right)^{w_{i}}\right)^{(1 / \theta)}\right)^{\theta}\right)^{p}\left(1-\left(1-\left(1-\left(1-\left(1-\left(d_{i} / t\right)\right)^{\theta}\right)^{w_{j}}\right)^{(1 / \theta)}\right)^{\theta}\right)^{q} . \\
& a_{4}^{\prime}=\left(1-\left(1-\left(1-\left(1-\left(a_{i} / t\right)^{\theta}\right)^{w_{i}}\right)^{(1 / \theta)}\right)^{\theta}\right)^{p}\left(1-\left(1-\left(1-\left(1-\left(a_{j} / t\right)^{\theta}\right)^{w_{j}}\right)^{(1 / \theta)}\right)^{\theta}\right)^{q}, \\
& b_{4}^{\prime}=\left(1-\left(1-\left(1-\left(1-\left(b_{i} / t\right)^{\theta}\right)^{w_{i}}\right)^{(1 / \theta)}\right)^{\theta}\right)^{p}\left(1-\left(1-\left(1-\left(1-\left(b_{j} / t\right)^{\theta}\right)^{w_{j}}\right)^{(1 / \theta)}\right)^{\theta}\right)^{q}, \\
& c_{4}
\end{aligned}
$$

\section{LIMADM Approach}

In this part, we will give an approach for MAGDM. In general, a MAGDM problem consists of the following parts: (1) alternative set: $\Xi=\left\{\Psi_{1}, \ldots, \Psi_{m}\right\}$; (2) attribute (criteria) set: $A=\left\{a_{1}, \ldots, a_{n}\right\} ;(3) \mathrm{WV}$ of attribute $W=\left(w_{1}, \ldots, w_{n}\right)^{T}$ satisfies $w_{i} \in[0,1]$ and $\sum_{i=1}^{n} w_{i}=1$; and (4) $D=\left\{D_{1}, D_{2}, \ldots, D_{p}\right\}$ is the set of DMs.

DMs evaluate the attribute value of alternative $\Psi_{i}$ under the attribute $a_{j}$ which can be expressed by LIVIFNs: $\gamma_{i j}^{k}=\left(\left[s_{a_{i j}^{k}}, s_{b_{i j}^{k}}\right],\left[s_{c_{i j}^{k}}, s_{d_{i j}^{k}}\right]\right)$. Then, an algorithm and process of MAGDM will be designed and given as follows:
Step 1: a revised decision matrix $\widehat{R}^{k}=\left(\widehat{\gamma}_{i j}^{k}\right)_{m \times n}$ is obtained by normalizing the original decision matrix $R$ in terms of the following equation:

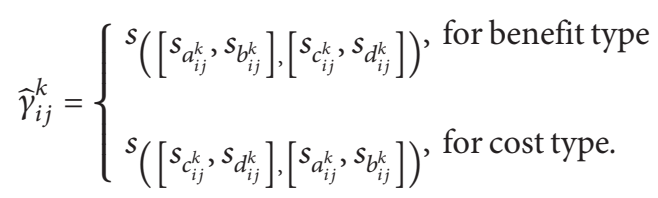

Step 2: all attribute values $\hat{\gamma}_{i j}^{k}(i=1, \ldots, m$; $j=1, \ldots, n ; k=1, \ldots, p ;)$ are aggregated to a 
comprehensive value $Z_{i}^{k}$ by the $G$-LIVIFWCHM ${ }^{p, q}$ operator.

Step 3: the supports are calculated:

$$
\begin{aligned}
& \operatorname{Sup}\left(Z_{i}^{k}, Z_{i}^{t}\right)=1-\left|K_{F}\left(Z_{i}^{k}\right)-K_{F}\left(Z_{i}^{t}\right)\right| \\
& K_{F}\left(Z_{i}^{k}\right)=\frac{\sqrt{\sum_{m=a}^{d}\left(m_{i}^{k}\right)^{2}+\left(a_{i}^{k}+c_{i}^{k}\right)^{2}+\left(b_{i}^{k}+d_{i}^{k}\right)^{2}}}{4} .
\end{aligned}
$$

Step 4: $T\left(Z_{i}^{k}\right)$ and weights $w_{i}^{k}$ are calculated:

$$
\begin{aligned}
T\left(Z_{i}^{k}\right) & =\sum_{t=1, k \neq t}^{p} \operatorname{Sup}\left(Z_{i}^{k}, Z_{i}^{t}\right), \\
w_{i}^{k} & =\frac{\lambda_{k}\left(1+T\left(Z_{i}^{k}\right)\right)}{\sum_{k=1}^{p} \lambda_{k}\left(1+T\left(Z_{i}^{k}\right)\right)},
\end{aligned}
$$

where $w_{i}^{k} \geq 0$, and $\sum_{k=1}^{p} w_{i}^{k}=1$.

Step 5: the $G$-LIVIFWCHM ${ }^{p, q}$ operator is used to obtain the collective preference values $Z_{i}$.

Step 6: the alternatives are ranked, and the desirable one is selected by equation (2).

The flowchart is shown in Figure 1.

\section{Case Analysis}

This example is from [12]. In the selection of companies for investment in the rural areas, there are four companies $\Psi_{1}, \Psi_{2}$, $\Psi_{3}$, and $\Psi_{4}$ as candidates. The following four attributes $\left(c_{1}, \ldots, c_{4}\right)$ should be considered: $c_{1}$ : project cost; $c_{2}$ : technical capability; $c_{3}$ : financial status; and $c_{4}$ : company background.

The experts use LVs $\mathcal{S}=\left\{s_{0}=\right.$ extremelypoor, $s_{1}=$ verypoor, $s_{2}=$ poor, $s_{3}=$ slightlypoor, $s_{4}=$ fair, $s_{5}=$ slightlygood, $s_{6}=$ good, $s_{7}=$ verygood, $s_{8}=$ extremelygood $\}$ to evaluate the companies in terms of LIVIFNs. The decision matrix can be found in Table 2 .

\subsection{Determining the Optimal Company}

Example 1. In this section, we use G- LIVIFWCHM ${ }^{p, q}$ operators to solve this MAGDM problem, where $q=1$ and $\theta=1$.

Let $\lambda=(0.243,0.514,0.243)$ be the $\mathrm{WV}$ of the three experts and $w=(0.4,0.25,0.2,0.15)$ be the WV of the attributes.

Step 1: since all attributes are of the same type, the normalization procedure is omitted.

Step 2: the proposed $G$ - LIVIFWCHM ${ }^{p, q}$ operator is employed to aggregate the evaluation values of each attribute into the integrated matrices $Z_{i}^{k}$, as shown in Table 3.

Step 3: the supports $S_{i}^{k t}=\operatorname{Sup}\left(Z_{i}^{k}, Z_{i}^{t}\right)$ are obtained according to equation (46): $S_{1}^{12}=S_{1}^{21}=0.9715$, $S_{1}^{13}=S_{1}^{31}=0.9663, S_{1}^{23}=S_{1}^{32}=0.9948, S_{2}^{12}=S_{2}^{21}=0.9602$, $S_{2}^{13}=S_{2}^{31}=0.9537, S_{2}^{23}=S_{2}^{32}=0.9936, S_{3}^{12}=S_{3}^{21}=0.9625$,
$S_{3}^{13}=S_{3}^{31}=0.9774, S_{3}^{23}=S_{3}^{32}=0.9851, S_{4}^{12}=S_{4}^{21}=0.9657$, $S_{4}^{13}=S_{4}^{31}=0.9640$, and $S_{4}^{23}=S_{4}^{32}=0.9982$.

Step 4: the supports $T_{i}^{k}=T\left(Z_{i}^{k}\right)$ and the weights $w_{i}^{k}$ are obtained according to equations (47) and (48):

$$
\begin{aligned}
T_{1}^{1} & =\sum_{t=1, t \neq 1}^{3} \operatorname{Sup}\left(Z_{1}^{1}, Z_{1}^{t}\right)=\operatorname{Sup}\left(Z_{1}^{1}, Z_{1}^{2}\right)+\operatorname{Sup}\left(Z_{1}^{1}, Z_{1}^{3}\right) \\
& =1.9378,
\end{aligned}
$$

$T_{1}^{2}=1.9663$

$T_{1}^{3}=1.9610$,

$T_{2}^{1}=1.9139$,

$T_{2}^{2}=1.9537$

$T_{2}^{3}=1.9473$,

$T_{3}^{1}=1.9399$,

$T_{3}^{2}=1.9477$,

$T_{3}^{3}=1.9625$,

$T_{4}^{1}=1.9297$,

$T_{4}^{2}=1.9640$,

$T_{4}^{3}=1.9622$

$\omega_{1}^{1}=\frac{\lambda_{1}\left(1+T_{1}^{1}\right)}{\sum_{k=1}^{3} \lambda_{k}\left(1+T_{1}^{k}\right)}$

Similarly, we have

$$
\begin{aligned}
& \omega_{1}^{2}=0.5154, \\
& \omega_{1}^{3}=0.2432, \\
& \omega_{2}^{1}=0.2406, \\
& \omega_{2}^{2}=0.5160, \\
& \omega_{2}^{3}=0.2434, \\
& \omega_{3}^{1}=0.2422, \\
& \omega_{3}^{2}=0.5137, \\
& \omega_{3}^{2}=0.2441, \\
& \omega_{4}^{1}=0.2409, \\
& \omega_{4}^{2}=0.5155, \\
& \omega_{3}^{2}=0.2436
\end{aligned}
$$




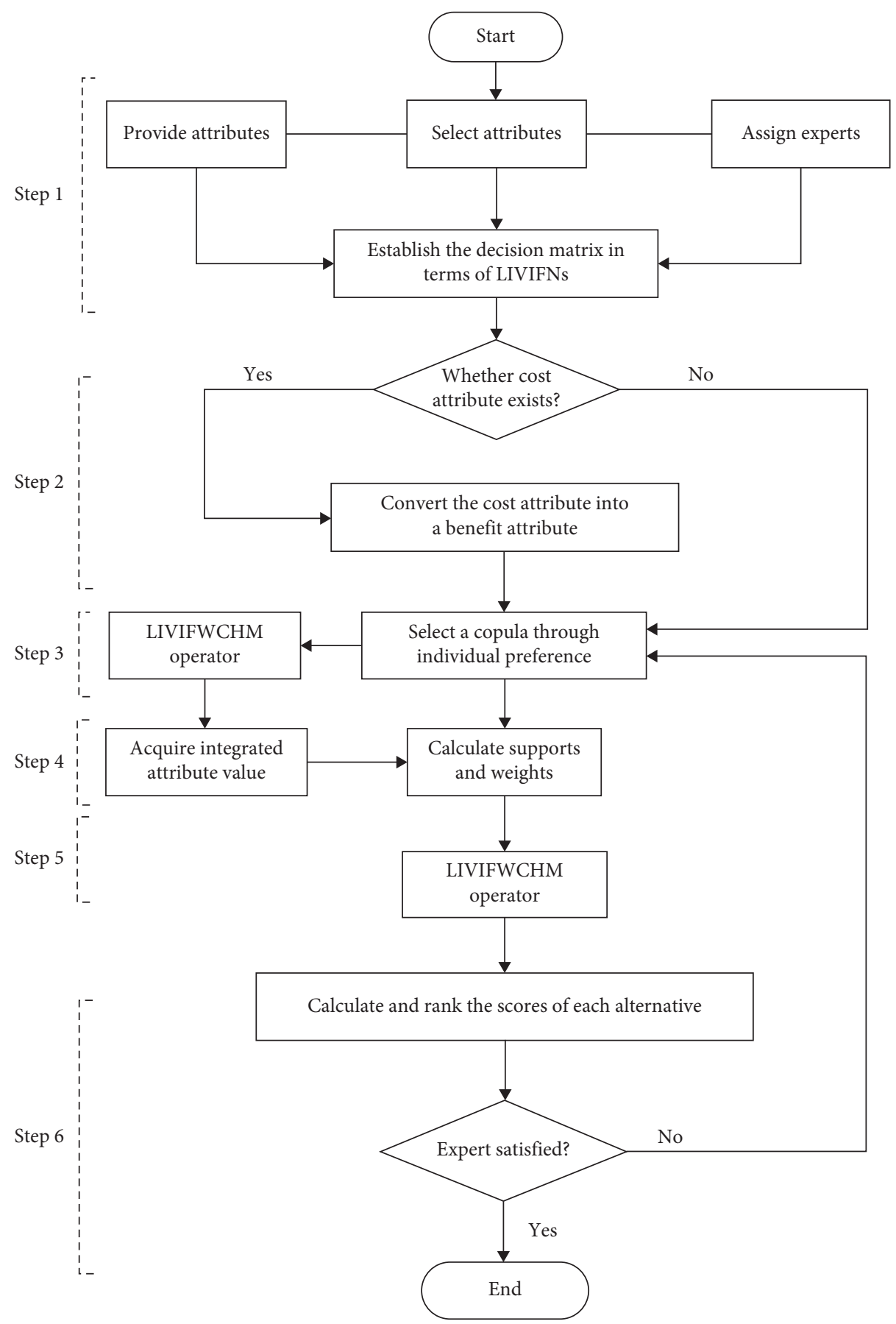

Figure 1: Flowchart of MAGDM.

Step 5: the collective preference values $Z_{i}$ are obtained according to the $G$ - LIVIFWCHM $^{p, q}$ operator:
Step 6: computing the score values of every alternative on the basis of Definition 2, we have

$$
\begin{aligned}
& Z_{1}=\left(\left[s_{3.3828}, s_{5.5831}\right],\left[s_{6.7725}, s_{7.1948}\right]\right), \\
& Z_{2}=\left(\left[s_{2.2704}, s_{4.4453}\right],\left[s_{6.9295}, s_{7.3946}\right]\right), \\
& Z_{3}=\left(\left[s_{3.3408}, s_{5.5389}\right],\left[s_{6.8444}, s_{7.2586}\right]\right), \\
& Z_{4}=\left(\left[s_{3.3786}, s_{4.4738}\right],\left[s_{7.0047}, s_{7.2643}\right]\right) .
\end{aligned}
$$

$$
\begin{aligned}
& S\left(\Psi_{1}\right)=0.7497, \\
& S\left(\Psi_{2}\right)=0.5979, \\
& S\left(\Psi_{3}\right)=0.6942 \\
& S\left(\Psi_{4}\right)=0.6458 .
\end{aligned}
$$


Table 2: Decision matrix $R^{k}(k=1,2,3)$.

\begin{tabular}{|c|c|c|c|c|c|}
\hline & & $c_{1}$ & $c_{2}$ & $c_{3}$ & $c_{4}$ \\
\hline$R^{1}$ & $\begin{array}{l}\Psi_{1} \\
\Psi_{2} \\
\Psi_{3} \\
\Psi_{4}\end{array}$ & $\begin{array}{l}\left(\left[s_{5}, s_{6}\right],\left[s_{1}, s_{2}\right]\right) \\
\left(\left[s_{3}, s_{5}\right],\left[s_{2}, s_{3}\right]\right) \\
\left(\left[s_{5}, s_{6}\right],\left[s_{1}, s_{2}\right]\right) \\
\left(\left[s_{4}, s_{5}\right],\left[s_{2}, s_{3}\right]\right)\end{array}$ & $\begin{array}{l}\left(\left[s_{4}, s_{6}\right],\left[s_{1}, s_{1}\right]\right) \\
\left(\left[s_{5}, s_{6}\right],\left[s_{1}, s_{2}\right]\right) \\
\left(\left[s_{5}, s_{6}\right],\left[s_{1}, s_{2}\right]\right) \\
\left(\left[s_{1}, s_{3}\right],\left[s_{3}, s_{4}\right]\right)\end{array}$ & $\begin{array}{r}\left(\left[s_{4}, s_{5}\right],\left[s_{2}, s_{3}\right]\right) \\
\left(\left[s_{2}, s_{4}\right],\left[s_{3}, s_{4}\right]\right) \\
\left(\left[s_{3}, s_{5}\right],\left[s_{2}, s_{3}\right]\right) \\
\left(\left[s_{3}, s_{5}\right],\left[s_{1}, s_{3}\right]\right)\end{array}$ & $\begin{array}{l}\left(\left[s_{6}, s_{7}\right],\left[s_{1}, s_{1}\right]\right) \\
\left(\left[s_{3}, s_{4}\right],\left[s_{2}, s_{3}\right]\right) \\
\left(\left[s_{3}, s_{5}\right],\left[s_{1}, s_{3}\right]\right) \\
\left(\left[s_{6}, s_{7}\right],\left[s_{1}, s_{1}\right]\right)\end{array}$ \\
\hline$R^{2}$ & $\begin{array}{l}\Psi_{1} \\
\Psi_{2} \\
\Psi_{3} \\
\Psi_{4}\end{array}$ & $\begin{array}{l}\left(\left[s_{2}, s_{4}\right],\left[s_{1}, s_{3}\right]\right) \\
\left(\left[s_{3}, s_{5}\right],\left[s_{1}, s_{3}\right]\right) \\
\left(\left[s_{3}, s_{4}\right],\left[s_{1}, s_{2}\right]\right) \\
\left(\left[s_{4}, s_{5}\right],\left[s_{1}, s_{2}\right]\right)\end{array}$ & $\begin{array}{l}\left(\left[s_{4}, s_{5}\right],\left[s_{1}, s_{2}\right]\right) \\
\left(\left[s_{1}, s_{2}\right],\left[s_{1}, s_{4}\right]\right) \\
\left(\left[s_{3}, s_{6}\right],\left[s_{1}, s_{2}\right]\right) \\
\left(\left[s_{3}, s_{3}\right],\left[s_{3}, s_{5}\right]\right)\end{array}$ & $\begin{array}{l}\left(\left[s_{4}, s_{5}\right],\left[s_{1}, s_{3}\right]\right) \\
\left(\left[s_{2}, s_{3}\right],\left[s_{3}, s_{4}\right]\right) \\
\left(\left[s_{2}, s_{5}\right],\left[s_{2}, s_{3}\right]\right) \\
\left(\left[s_{3}, s_{3}\right],\left[s_{2}, s_{3}\right]\right)\end{array}$ & $\begin{array}{l}\left(\left[s_{3}, s_{6}\right],\left[s_{1}, s_{2}\right]\right) \\
\left(\left[s_{3}, s_{5}\right],\left[s_{1}, s_{3}\right]\right) \\
\left(\left[s_{3}, s_{4}\right],\left[s_{2}, s_{3}\right]\right) \\
\left(\left[s_{4}, s_{6}\right],\left[s_{1}, s_{1}\right]\right)\end{array}$ \\
\hline$R^{3}$ & $\begin{array}{l}\Psi_{1} \\
\Psi_{2} \\
\Psi_{3} \\
\Psi_{4}\end{array}$ & $\begin{array}{l}\left(\left[s_{2}, s_{4}\right],\left[s_{1}, s_{2}\right]\right) \\
\left(\left[s_{1}, s_{4}\right],\left[s_{2}, s_{3}\right]\right) \\
\left(\left[s_{2}, s_{3}\right],\left[s_{1}, s_{5}\right]\right) \\
\left(\left[s_{3}, s_{4}\right],\left[s_{2}, s_{3}\right]\right) \\
\end{array}$ & $\begin{array}{l}\left(\left[s_{2}, s_{3}\right],\left[s_{1}, s_{4}\right]\right) \\
\left(\left[s_{4}, s_{5}\right],\left[s_{1}, s_{2}\right]\right) \\
\left(\left[s_{3}, s_{5}\right],\left[s_{1}, s_{2}\right]\right) \\
\left(\left[s_{1}, s_{2}\right],\left[s_{3}, s_{4}\right]\right) \\
\end{array}$ & $\begin{array}{l}\left(\left[s_{3}, s_{5}\right],\left[s_{2}, s_{3}\right]\right) \\
\left(\left[s_{2}, s_{4}\right],\left[s_{1}, s_{3}\right]\right) \\
\left(\left[s_{3}, s_{5}\right],\left[s_{1}, s_{3}\right]\right) \\
\left(\left[s_{3}, s_{5}\right],\left[s_{1}, s_{2}\right]\right) \\
\end{array}$ & $\begin{array}{l}\left(\left[s_{5}, s_{7}\right],\left[s_{1}, s_{1}\right]\right) \\
\left(\left[s_{3}, s_{4}\right],\left[s_{2}, s_{4}\right]\right) \\
\left(\left[s_{3}, s_{5}\right],\left[s_{2}, s_{3}\right]\right) \\
\left(\left[s_{5}, s_{6}\right],\left[s_{1}, s_{1}\right]\right) \\
\end{array}$ \\
\hline
\end{tabular}

TABLE 3: Integrated decision matrix $Z_{i}^{k}$.

\begin{tabular}{llll}
\hline & $Z^{1}$ & $Z^{2}$ & $Z^{3}$ \\
\hline$Z_{1}$ & $\left(\left[s_{1.6304}, s_{2.3639}\right],\left[s_{4.9640}, s_{5.4021}\right]\right)$ & $\left(\left[s_{0.9388}, s_{1.6541}\right],\left[s_{4.7882}, s_{6.0148}\right]\right)$ & $\left(\left[s_{0.8144}, s_{1.6166}\right],\left[s_{4.9640}, s_{5.8864}\right]\right)$ \\
$Z_{2}$ & $\left(\left[s_{1.0577}, s_{1.7387}\right],\left[s_{5.5472}, s_{6.1952}\right]\right)$ & $\left(\left[s_{0.6849}, s_{1.3032}\right],\left[s_{5.0710}, s_{6.4657}\right]\right)$ & $\left(\left[s_{0.6894}, s_{1.3951}\right],\left[s_{5.2463}, s_{6.1729}\right]\right)$ \\
$Z_{3}$ & $\left(\left[s_{1.4590}, s_{2.1252}\right],\left[s_{4.9640}, s_{5.8748}\right]\right)$ & $\left(\left[s_{0.8296}, s_{1.6500}\right],\left[s_{5.1007}, s_{5.8748}\right]\right)$ & $\left(\left[s_{0.7574}, s_{1.4120}\right],\left[s_{4.9224}, s_{6.4236}\right]\right)$ \\
$Z_{3}$ & $\left(\left[s_{1.1480}, s_{1.7950}\right],\left[s_{5.4708}, s_{6.1195}\right]\right)$ & $\left(\left[s_{1.1087}, s_{1.4725}\right],\left[s_{5.3195}, s_{5.9689}\right]\right)$ & $\left(\left[s_{0.8829}, s_{1.3765}\right],\left[s_{5.4708}, s_{5.9967}\right]\right)$ \\
\hline
\end{tabular}

The rank of alternatives is $\Psi_{1}>\Psi_{3}>\Psi_{4}>\Psi_{2}$, and so, $\Psi_{1}$ is the best alternative.

The ordering results of alternatives use other ECs proposed in the present work which are listed in Table 4.

5.2. Sensitivity Analysis. The following two aspects reflect the flexibility of this method: firstly, DMs can select different types of ECs and ECCs with parameter $\theta$; secondly, the HM operator contains two important parameters, which can reflect the correlation between attributes. Therefore, different ranking results may be obtained according to different parameters.

In the following, the influence of parameters $p, q$, and $\theta$ on the results will be analyzed. Without loss of generality, the following analysis adopts the $G-$ LIVIFWCHM $^{p, q}$ operator. Firstly, we assign different values to $\theta$ with fixed $p$ and $q$, and the results are listed in Table 5. In addition, we explore the effect of parameters $p$ and $q$ on the ultimate ranking results which can be found in Table 6 and Figures 2-9.

From Table 5, we can find out that when $p$ and $q$ are fixed, $\theta$ has little influence on the sorting result. From Figures 2-9 and Table 6, it is easy to derive the following conclusions: (1) the scores and ranking order will be different with respect to different parameters $p$ and $q$. (2) The optimal candidate will change when $\theta$ is small, and the absolute value of $p$ minus $q$ is large. (3) The best and worst alternatives are always the same when $\theta \geq 2$, and $q=1$, or $q=1$.

In application, the larger the value of $p$ or $q$, the more prominent the interaction between attributes, and if one of the parameters is zero, the relationship between attributes is not considered. For the actual applications, we can choose a simple integral number for $p$ and $q$ to simplify the process.
5.3. Comparative Analysis. In the following, the proposed approach will be analyzed and compared with other existing methods.

Example 2. This example is to select a new management information system. There are four alternatives $A_{i}(i=1,2,3,4)$ to be considered. Following this, there are four attributes $C_{1}, C_{2}, C_{3}$, and $C_{4}$ to be evaluated by three DMs using LIVIFI, where $C_{1}$ : the costs; $C_{2}$ : the reliability of software development from outsourcing enterprise; $C_{3}$ : the contribution to the enterprise performance; and $C_{4}$ : the effort to transition to a new system from the old systems.

For the decision matrices, see Tables III-V in [50]. The score values and rankings of alternatives are separately displayed in Table 7. From it, we can draw a conclusion that the orders are almost the same, and the optimal selections are all $A_{4}$, so we can see the proposed approach is workable and efficient.

Example 3. This is a MADM problem which is from [14]. In the example, a company wants to establish a new subsidiary on four potential sites $\Psi=\left\{\Psi_{1}, \Psi_{2}, \Psi_{3}, \Psi_{4}\right\}$, and there are five attributes $C=\left\{c_{1}, c_{2}, c_{3}, c_{4}, c_{5}\right\}$ that affect decisionmaking, and the weight of attributes is $\omega=\{0.2,0.25,0.15,0.18,0.22\}$. The evaluation value is expressed by the IVLIFN which is shown in Table 8 . The proposed method used the LIVIFWCHM operator (see equation (21)), and also, the same score function proposed by Garg and Kumar [12] (equation (2)) was used for easy comparison. The comparison results with the recent existing work are listed in Table 9.

In the comparison, the WA operator in [12] and PWA operator in [13] were chosen which ignored the interaction 
TABLE 4: The ordering results of alternatives using other different copulas.

\begin{tabular}{lccr}
\hline Type of copulas & Parameters & Score index of $\Psi_{i}(i=1,2,3,4)$ & Ranking order \\
\hline Clayton & $\theta=1$ & $1.3856,0.9479,1.1720,1.0664$ & $\Psi_{1}>\Psi_{3}>\Psi_{4}>\Psi_{2}$ \\
Frank & $\theta=1$ & $0.9278,0.7177,0.8458,0.7839$ & $\Psi_{1}>\Psi_{3}>\Psi_{4}>\Psi_{2}$ \\
Ali-Mikhail-Haq & $\theta=-1$ & $1.6916,1.6132,1.6703,1.6391$ & $\Psi_{1}>\Psi_{3}>\Psi_{4}>\Psi_{2}$ \\
Joe & $\theta=1$ & $0.7704,0.6149,0.7154,0.6653$ & $\Psi_{1}>\Psi_{3}>\Psi_{4}>\Psi_{2}$ \\
\hline
\end{tabular}

TABLE 5: The influence of parameter $\theta$ on the rank of alternatives $(p=q=1)$.

\begin{tabular}{lrr}
\hline$\theta$ & Score index of $\Psi_{i}(i=1,2,3,4)$ & Ranking order \\
\hline$\theta=2$ & $2.3003,1.8367,2.0952,2.0216$ & $\Psi_{1}>\Psi_{3}>\Psi_{4}>\Psi_{2}$ \\
$\theta=3$ & $3.2580,2.6363,2.9632,2.9331$ & $\Psi_{1}>\Psi_{3}>\Psi_{4}>\Psi_{2}$ \\
$\theta=5$ & $4.2744,3.5199,3.8836,3.9829$ & $\Psi_{1}>\Psi_{3}>\Psi_{4}>\Psi_{2}$ \\
$\theta=10$ & $5.3432,4.4847,4.8243,5.1902$ & $\Psi_{1}>\Psi_{3}>\Psi_{4}>\Psi_{2}$ \\
\hline
\end{tabular}

TABLE 6: The influence of parameter $q$ on the rank of alternatives $(\theta=1)$.

\begin{tabular}{|c|c|c|c|}
\hline$p$ & $q$ & Score index of $\Psi_{i}(i=1,2,3,4)$ & Ranking order \\
\hline \multirow{3}{*}{$p=0$} & $q=1$ & $0.8477,0.6880,0.8302,0.6917$ & $\Psi_{1}>\Psi_{3}>\Psi_{4}>\Psi_{2}$ \\
\hline & $q=2$ & $0.9082,0.7696,0.9108,0.7984$ & $\Psi_{1}>\Psi_{3}>\Psi_{4}>\Psi_{2}$ \\
\hline & $q=5$ & $1.0758,0.9895,1.1331,1.0987$ & $\Psi_{3}>\Psi_{4}>\Psi_{1}>\Psi_{2}$ \\
\hline \multirow{4}{*}{$p=1$} & $q=0$ & $0.6644,0.5043,0.5727,0.5897$ & $\Psi_{1}>\Psi_{4}>\Psi_{3}>\Psi_{2}$ \\
\hline & $q=1$ & $0.7497,0.5979,0.6942,0.6458$ & $\Psi_{1}>\Psi_{3}>\Psi_{4}>\Psi_{2}$ \\
\hline & $q=2$ & $0.8285,0.6897,0.7972,0.7386$ & $\Psi_{3}>\Psi_{1}>\Psi_{4}>\Psi_{2}$ \\
\hline & $q=5$ & $1.0126,0.9099,1.0362,1.0089$ & $\Psi_{3}>\Psi_{1}>\Psi_{4}>\Psi_{2}$ \\
\hline \multirow{4}{*}{$p=2$} & $q=0$ & $0.7219,0.5713,0.6485,0.6551$ & $\Psi_{1}>\Psi_{4}>\Psi_{3}>\Psi_{2}$ \\
\hline & $q=1$ & $0.7755,0.6379,0.7321,0.6983$ & $\Psi_{1}>\Psi_{3}>\Psi_{4}>\Psi_{2}$ \\
\hline & $q=2$ & $0.8367,0.7129,0.8155,0.7740$ & $\Psi_{1}>\Psi_{3}>\Psi_{4}>\Psi_{2}^{2}$ \\
\hline & $q=5$ & $0.9978,0.9034,1.0237,1.0031$ & $\Psi_{3}>\Psi_{4}>\Psi_{1}>\Psi_{2}$ \\
\hline
\end{tabular}

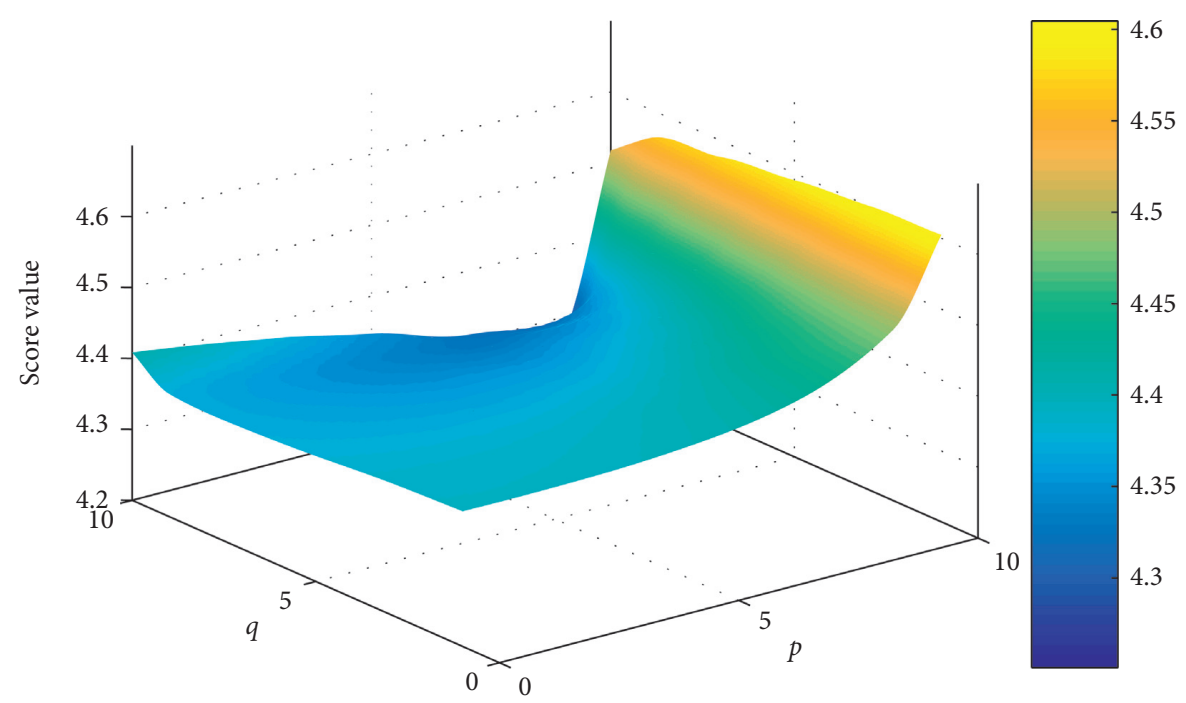

Figure 2: Scores of $A_{1}$ when $p, q \in[0,10](\theta=5)$.

between attributes. However, the YWMSM operator in [14] and WPMM operator in [15] considered the interaction between attributes. As can be seen from Table 9, the ranking order of the proposed method is exactly the same with Liu and Qin [14] and Qin [15]. Therefore, it can be concluded that the method proposed in this paper is feasible and effective for dealing with MAGDM problems based on LIVIFNs. In addition, the ranking results of the WA operator and PWA operator are different from those of other methods. The reason is that the former methods assume that all attributes are independent. Furthermore, compared with the YWMSM operator in [14] and WPMM operator in [15], the proposed method is based on ECs and ECCs, which have 5 different generator functions, so it can provide DMs more options. Therefore, this method is more flexible. 


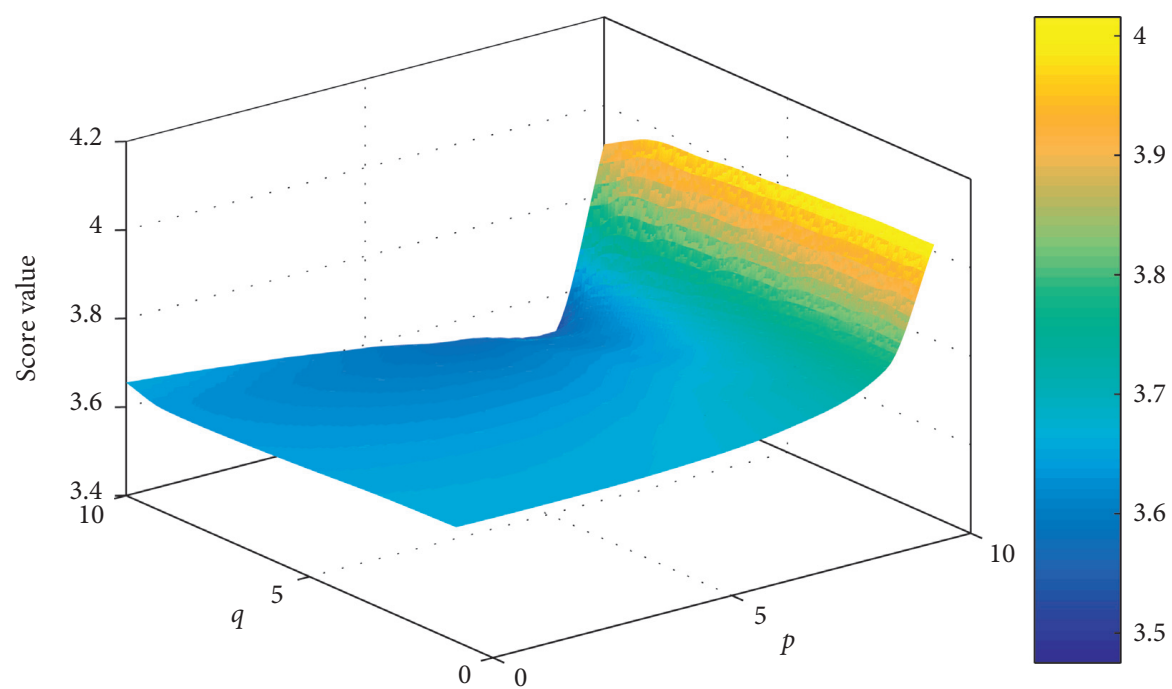

Figure 3: Scores of $A_{2}$ when $p, q \in[0,10](\theta=5)$.

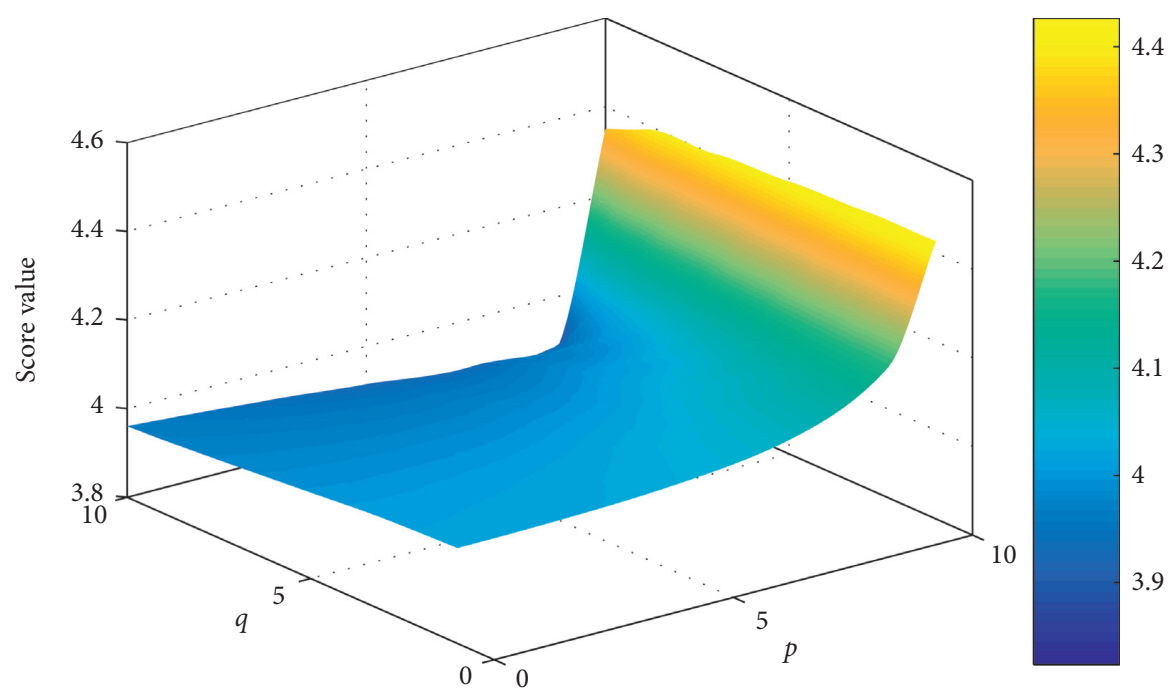

Figure 4: Scores of $A_{3}$ when $p, q \in[0,10](\theta=5)$.

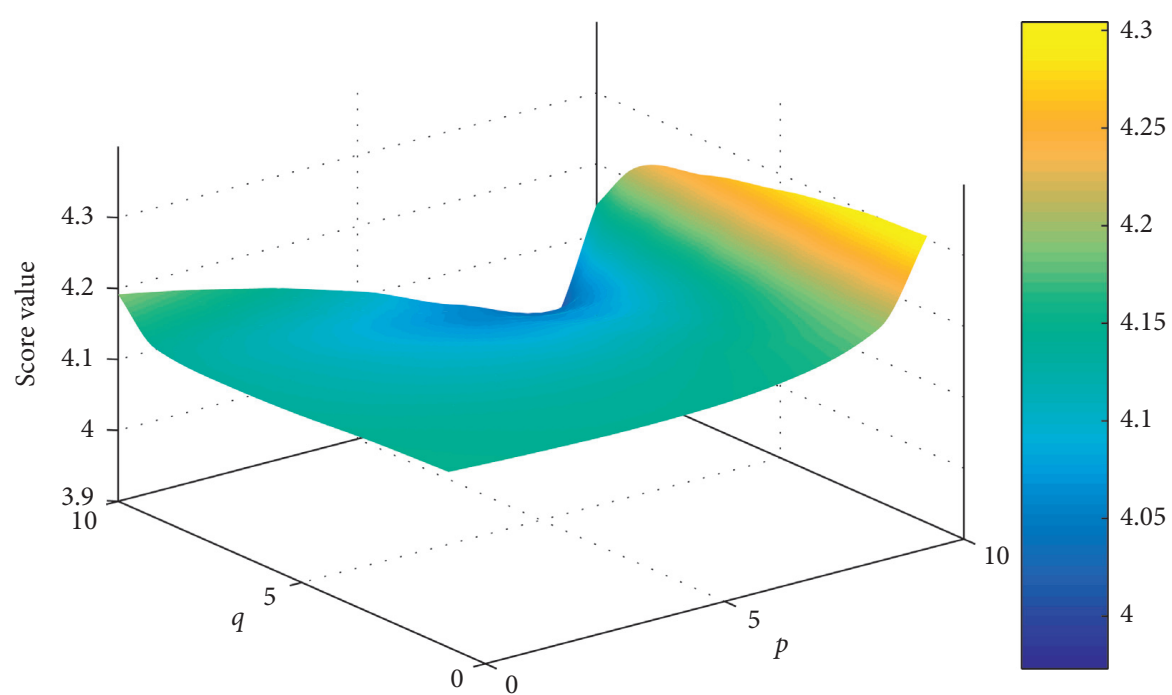

Figure 5: Scores of $A_{4}$ when $p, q \in[0,10](\theta=5)$. 

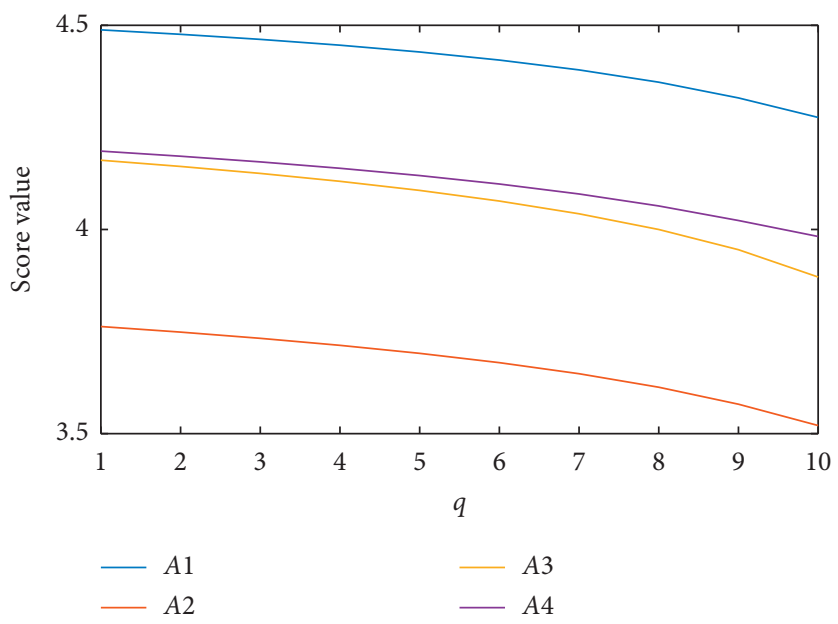

Figure 6: Scores of $A_{i}(i=1,2,3,4)$ when $p=1$ and $q \in[0,10](\theta=5)$.
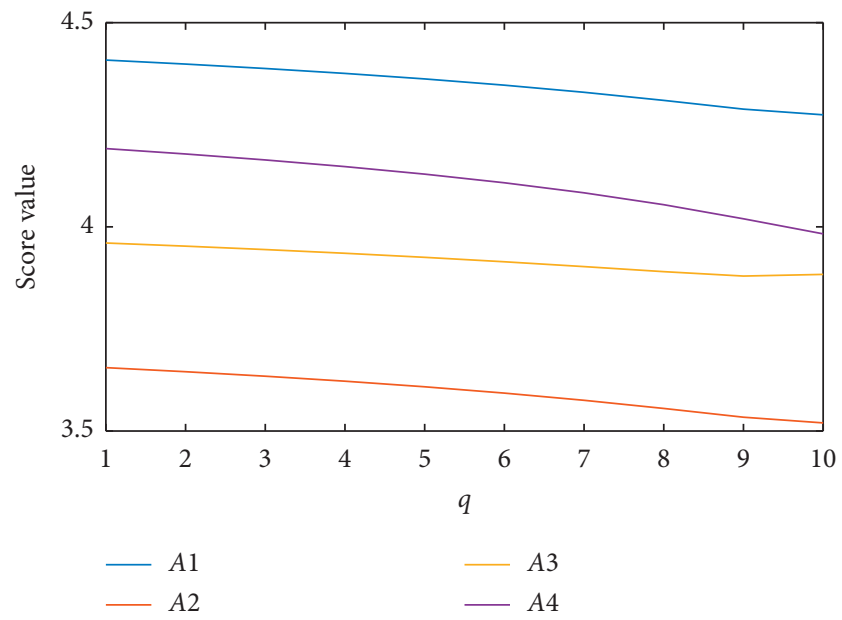

Figure 7: Scores of $A_{i}(i=1,2,3,4)$ when $q=1$ and $p \in[0,10](\theta=5)$.

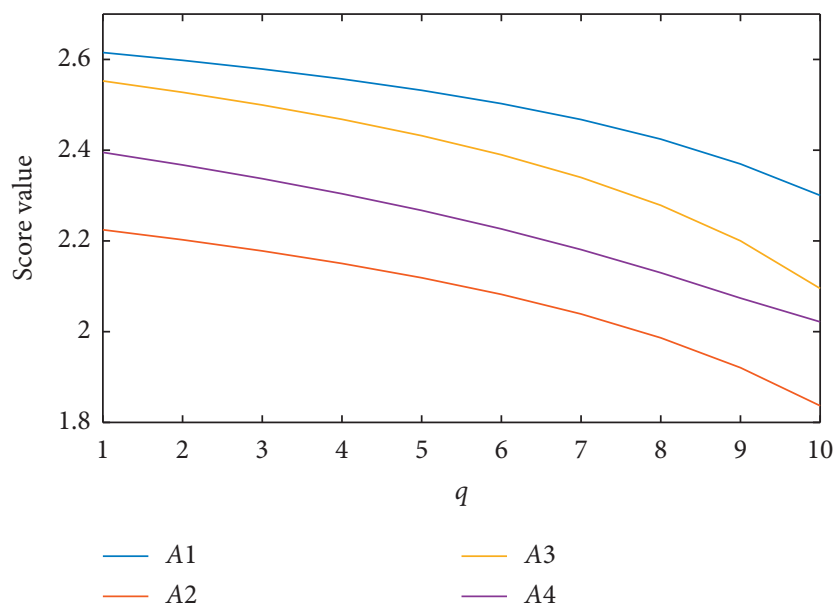

Figure 8: Scores of $A_{i}(i=1,2,3,4)$ when $p=1$ and $q \in[0,10](\theta=2)$. 


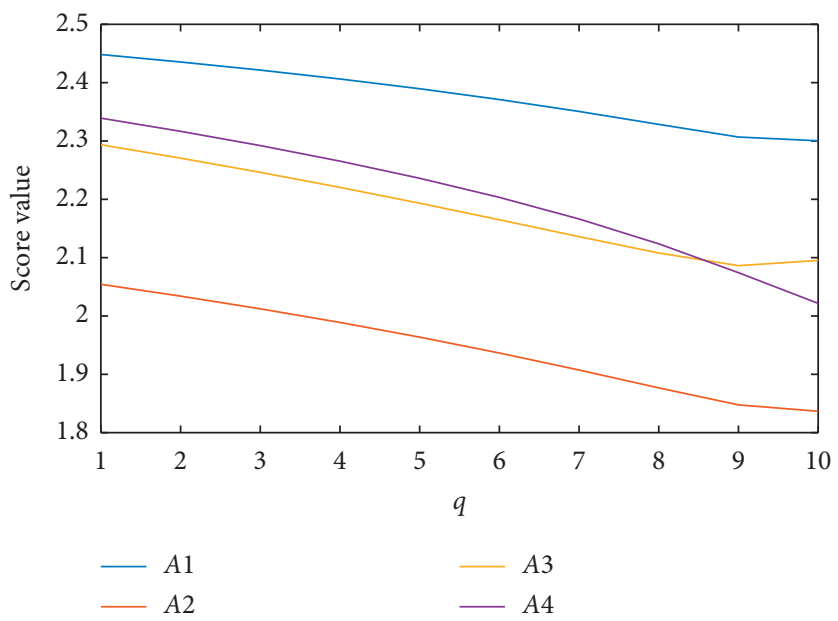

Figure 9: Scores of $A_{i}(i=1,2,3,4)$ when $q=1$ and $p \in[0,10](\theta=2)$.

TABLE 7: Comparison with existing approaches for Example 2.

\begin{tabular}{lccc}
\hline Methods & Parameters & Score index of $A_{i}(i=1,2,3,4)$ & Ranking order \\
\hline He et al. [51] & $\lambda=2$ & $0.0167,0.0931,0.0808,0.2676$ & $A_{4}>A_{3}>A_{2}>A_{1}$ \\
Yu and Wu [44] & $p=2, q=2$ & $0.0740,0.1078,0.2868,0.3025$ & $A_{4}>A_{3}>A_{2}>A_{1}$ \\
Liu [50] & $p=2, q=2$ & $0.0719,0.1090,0.2846,0.3022$ & $A_{4}>A_{3}>A_{2}>A_{1}$ \\
Gumbel type & $\theta=1, p=q=1$ & $0.0565,0.0598,0.0721,0.0777$ & $A_{4}>A_{3}>A_{2}>A_{1}$ \\
Clayton type & $\theta=1, p=q=1$ & $0.0712,0.0837,0.0811,0.1026$ & $A_{4}>A_{2}>A_{3}>A_{1}$ \\
Frank type & $\theta=1, p=q=1$ & $0.0656,0.0705,0.0850,0.0912$ & $A_{4}>A_{3}>A_{2}>A_{1}$ \\
Ali-Mikhail-Haq type & $\theta=-1, p=q=1$ & $0.1884,0.1904,0.1969,0.1981$ & $A_{4}>A_{3}>A_{2}>A_{1}$ \\
Joe type & $\theta=1, p=q=1$ & $0.0590,0.0622,0.0770,0.0821$ & $A_{4}>A_{3}>A_{2}>A_{1}$ \\
\hline
\end{tabular}

TABle 8: Decision matrix $R$.

\begin{tabular}{|c|c|c|c|c|c|}
\hline & $c_{1}$ & $c_{2}$ & $c_{3}$ & $c_{4}$ & $c_{5}$ \\
\hline$\Psi_{1}$ & $\left(\left[s_{6}, s_{7}\right],\left[s_{1}, s_{1}\right]\right)$ & $\left(\left[s_{5}, s_{6}\right],\left[s_{1}, s_{2}\right]\right)$ & $\left(\left[s_{4}, s_{5}\right],\left[s_{1}, s_{3}\right]\right)$ & $\left(s_{6},\left[s_{7}\right]\right),\left[s_{1}, s_{1}\right]$ & $\left(\left[s_{5}, s_{6}\right],\left[s_{1}, s_{2}\right]\right)$ \\
\hline$\Psi_{2}$ & $\left(\left[s_{4}, s_{5}\right],\left[s_{1}, s_{2}\right]\right)$ & $\left(\left[s_{5}, s_{7}\right],\left[s_{1}, s_{1}\right]\right)$ & $\left(\left[s_{5}, s_{6}\right],\left[s_{1}, s_{2}\right]\right)$ & $\left(\left[s_{5}, s_{6}\right],\left[s_{1}, s_{2}\right]\right)$ & $\left(\left[s_{6}, s_{7}\right],\left[s_{1}, s_{1}\right]\right)$ \\
\hline$\Psi_{3}$ & $\left(\left[s_{5}, s_{6}\right],\left[s_{1}, s_{2}\right]\right)$ & $\left(\left[s_{4}, s_{5}\right],\left[s_{2}, s_{3}\right]\right)$ & $\left(\left[s_{6}, s_{7}\right],\left[s_{1}, s_{1}\right]\right)$ & $\left(\left[s_{5}, s_{6}\right],\left[s_{1}, s_{2}\right]\right)$ & $\left(\left[s_{3}, s_{4}\right],\left[s_{3}, s_{4}\right]\right)$ \\
\hline$\Psi_{4}$ & $\left(\left[s_{4}, s_{5}\right],\left[s_{2}, s_{3}\right]\right)$ & $\left(\left[s_{6}, s_{7}\right],\left[s_{1}, s_{1}\right]\right)$ & $\left(\left[s_{4}, s_{5}\right],\left[s_{2}, s_{3}\right]\right)$ & $\left(\left[s_{4}, s_{6}\right],\left[s_{1}, s_{2}\right]\right)$ & $\left(\left[s_{3}, s_{4}\right],\left[s_{3}, s_{4}\right]\right)$ \\
\hline
\end{tabular}

TABle 9: Comparison with existing approaches for Example 3.

\begin{tabular}{|c|c|c|c|c|}
\hline Methods & Used AOs & Parameters & Score index of $\Psi(i=1,2,3,4)$ & Ranking order \\
\hline Garg and Kumar [12] & WA & None & 6.26216 .27095 .61115 .5823 & $\Psi_{2}>\Psi_{1}>\Psi_{3}>\Psi_{4}$ \\
\hline Garg and Kumar [13] & PWA & None & 6.14076 .16205 .33265 .2541 & $\Psi_{2}>\Psi_{1}>\Psi_{3}>\Psi_{4}$ \\
\hline Liu and Qin [14] & YWMSM & $k=3$ & 6.10346 .06115 .51965 .1819 & $\Psi_{1}>\Psi_{2}>\Psi_{3}>\Psi_{4}$ \\
\hline Qin [15] & WPMM & $Q=(1,2,3,0,0)$ & 6.15846 .11575 .56375 .3056 & $\Psi_{1}>\Psi_{2}>\Psi_{3}>\Psi_{4}$ \\
\hline The proposed method & WCHM & $\theta=1$ and $p=q=2$ & 6.11016 .07915 .36375 .2688 & $\Psi_{1}>\Psi_{2}>\Psi_{3}>\Psi_{4}$ \\
\hline
\end{tabular}

In the following, the proposed approach will be analyzed and compared with other existing method approaches:

(1) Chen et al.'s LIFWA operator [10], Zhang's LIFWA operator [52], and Liu and Wang's ILIFWA operator [53] are all based on the LIFS. In our proposed method, when $t=1, \theta=1, p=1$, and $q=0$ and only $s_{(a+b) / 2}$ and $s_{(c+d) / 2}$ are considered, LIVIFWCHM $^{p, q}$ reduces to LIFWCHM ${ }^{p, q}$. In those methods mentioned above, the operational rules are based on algebraic TN and algebraic TC, which are special forms of EC and ECC. So, our method can also be applied to intuitionistic fuzzy DMPs. Therefore, our proposed method is effective and feasible. Furthermore, the proposed approach will provide more choice for the decision maker in real DMPs.

(2) Compared with Tao et al.'s method [36], if $t=1, p=$ 1 , and $q=0$ and only $s_{(a+b) / 2}$ and $s_{(c+d) / 2}$ are considered, LIFWCHM $^{p, q}$ reduces to IFCAA $\omega$. Therefore, compared with $\operatorname{IFCAA}_{\omega}$ [36], the proposed method is the generalization of Tao et al. 
TABLE 10: The characteristic comparison of different AOs.

\begin{tabular}{lcccc}
\hline AOs & Capture correlation among attributes & Generalization & Flexibility & Deal with LIVIFNs \\
\hline Chen et al. [10] & No & No & No & No \\
Zhang [52] & No & Yes & No & No \\
Liu and Wang [53] & Yes & No & No & No \\
Garg and Kumar [12] & No & Yes & Yes & No \\
Tao et al. [36] & No & Yes & No & Yes \\
Liu and Qin [14] & Yes & Yes & No & Yes \\
Qin [15] & Yes & Yes & Yes & Yes \\
The proposed method & Yes & & \\
\hline
\end{tabular}

(3) Compared to the method of Garg and Kumar and Liu and Qin $[12,14]$, the proposed method is more universal and flexible. Five aggregation functions can be regarded through assigning diverse copulas to them; also, parameters $\theta, p$, and $q$ can be selected according to decision maker's attitude.

(4) In [15] Qin combined the MM operator and the PA operator under the ATT operations. Compared with our method, Qin considered the interconnection of diverse attributes, but the designed method is based on ATT which is the special form of ECs and ECCs.

A detailed comparative analysis for the aforementioned approaches is displayed in Table 10 .

\section{Conclusions}

In this paper, we propose a LIVIFWCHM operator to deal with MAGDM problems under LIVIFI. We establish a new version of copulas and cocopulas and several universal operational laws of LIVIFNs and study some special instances of them based on dissimilar copulas. Then, we give the generalized expression of the LIVIFWCHM operator and explore several characteristics and five specific expressions of the LIVIFWCHM operator. On this basis, we bring forward an approach to solve MAGDM problems based on the LIVIFN. Then, a detailed numerical example has been given to show how it works, and a set of experiments have been carried out to verify the efficacy and superiority of the propounded approach. The results also show that the proposed method is more general and flexible and can consider the correlation between attributes. In future, we shall focus, especially, on the correlation between attributes and incomplete attribute information, as well as the large-scale decision-making algorithm based on linguistic assessment theory and methodology.

\section{Data Availability}

The data used to support the findings of this study are included within the article.

\section{Conflicts of Interest}

The authors declare that they have no conflicts of interest.

\section{Acknowledgments}

This work was supported by the Scientific Research Project of Department of Education of Sichuan Province (nos.
17ZB0220 and 18ZA0273), Scientific Research Innovation Team of Neijiang Normal University (no. 18TD008), the Application Basic Research Plan Project of Sichuan Province (no. 2017JY0199), Project of Sichuan Provincial Education Department (no. JG2018-736), and Scientific Research Project of Neijiang Normal University (no. 2019YZ06).

\section{References}

[1] A. Celotto, V. Loia, and S. Senatore, "Fuzzy linguistic approach to quality assessment model for electricity network infrastructure," Information Sciences, vol. 304, pp. 1-15, 2015.

[2] L. A. Zadeh, "The concept of a linguistic variable and its application to approximate reasoning-I," Information Sciences, vol. 8, no. 3, pp. 199-249, 1975.

[3] Z. Xu, Uncertain Multi-Attribute Decision Making: Methods and Applications, Springer, Berlin, Germany, 2015.

[4] Z. Xu, "A method based on linguistic aggregation operators for group decision making with linguistic preference relations * 1," Information Sciences, vol. 166, no. 1-4, pp. 19-30, 2004.

[5] Z. $\mathrm{Xu}$, "A note on linguistic hybrid arithmetic averaging operator in multiple attribute group decision making with linguistic information," Group Decision and Negotiation, vol. 15, no. 6, pp. 593-604, 2006.

[6] X. Z. Gou, H. Liao, and F. Herrera, "Multiple criteria decision making based on distance and similarity measures under double hierarchy hesitant fuzzy linguistic environment," Computers \& Industrial Engineering, vol. 126, pp. 516-530, 2018.

[7] D. Xu, X. Chen, and D. Peng, "Distance measures for hesitant fuzzy linguistic sets and their applications in multiple criteria decision making," International Journal of Fuzzy Systems, vol. 20, no. 7, pp. 2111-2121, 2018.

[8] F. Jin, Z. Ni, L. Pei et al., "A decision support model for group decision making with intuitionistic fuzzy linguistic preferences relations," Neural Computing and Applications, vol. 31, no. S2, pp. 1103-1124, 2019.

[9] J.-q. Wang, Y. Yang, and L. Li, "Multi-criteria decisionmaking method based on single-valued neutrosophic linguistic Maclaurin symmetric mean operators," Neural Computing and Applications, vol. 30, no. 5, pp. 1529-1547, 2018.

[10] Z. Chen, P. Liu, and Z. Pei, “An approach to multiple attribute group decision making based on linguistic intuitionistic fuzzy numbers," International Journal of Computational Intelligence Systems, vol. 8, no. 4, pp. 747-760, 2015.

[11] H. Garg, "Linguistic Pythagorean fuzzy sets and its applications in multiattribute decision-making process," International Journal of Intelligent Systems, vol. 33, no. 6, pp. 1234-1263, 2018. 
[12] H. Garg and K. Kumar, "Linguistic interval-valued atanassov intuitionistic fuzzy sets and their applications to group decision making problems," IEEE Transactions on Fuzzy Systems, vol. 27, no. 12, pp. 2302-2311, 2019.

[13] K. Kumar and H. Garg, "Prioritized linguistic interval-valued aggregation operators and their applications in group decision-making problems," Mathematics, vol. 6, no. 10, p. 209, 2018.

[14] P. Liu and X. Qin, "A new decision-making method based on interval-valued linguistic intuitionistic fuzzy information," Cognitive Computation, vol. 11, no. 1, pp. 125-144, 2019.

[15] Y. Qin, "Linguistic interval-valued intuitionistic fuzzy archimedean power muirhead mean operators for multiattribute group decision-making," Complexity, vol. 2020, Article ID 2373762, 28 pages, 2020.

[16] W. Xu, X. Shang, J. Wang, and W. Li, “A novel approach to multi-attribute group decision-making based on intervalvalued intuitionistic fuzzy power Muirhead mean," Symmetry, vol. 11, no. 3, p. 441, 2019.

[17] S. Xian, W. Y. Yin, and Y. Xiao, "Intuitionistic fuzzy intervalvalued linguistic entropic combined weighted averaging operator for linguistic group decision making," International Journal of Intelligent Systems, vol. 33, no. 2, pp. 444-460, 2018.

[18] K. Du and H. Yuan, "Interval-valued intuitionistic 2-tuple linguistic Bonferroni mean operators and their applications in multi-attribute group decision making," International Journal of Fuzzy Systems, vol. 21, no. 8, pp. 2373-2391, 2019.

[19] Y. Liu, J. Liu, and Y. Qin, "Pythagorean fuzzy linguistic Muirhead mean operators and their applications to multiattribute decision-making," International Journal of Intelligent Systems, vol. 35, no. 2, 300 pages, 2019.

[20] L. Wu, G. Wei, J. Wu, and C. Wei, "Some interval-valued intuitionistic fuzzy Dombi heronian mean operators and their application for evaluating the ecological value of forest ecological tourism demonstration areas," International Journal of Environmental Research and Public Health, vol. 17, no. 3, p. 829, 2020.

[21] L. Wu, J. Wang, and H. Gao, "Models for competiveness evaluation of tourist destination with some interval-valued intuitionistic fuzzy Hamy mean operators," Journal of Intelligent \& Fuzzy Systems, vol. 36, no. 6, pp. 5693-5709, 2019.

[22] J. Lu and C. Wei, "TODIM method for performance appraisal on social-integration-based rural reconstruction with interval-valued intuitionistic fuzzy information," Journal of Intelligent \& Fuzzy Systems, vol. 37, no. 2, pp. 1731-1740, 2019.

[23] L. Wu, H. Gao, and C. Wei, "VIKOR method for financing risk assessment of rural tourism projects under intervalvalued intuitionistic fuzzy environment," Journal of Intelligent \& Fuzzy Systems, vol. 37, no. 2, pp. 2001-2008, 2019.

[24] H. Garg and K. Kumar, "A novel exponential distance and its based TOPSIS method for interval-valued intuitionistic fuzzy sets using connection number of SPA theory," Artificial Intelligence Review, vol. 53, no. 1, pp. 595-624, 2020.

[25] H. Garg and K. Kumar, "Power geometric aggregation operators based on connection number of set pair analysis under intuitionistic fuzzy environment," A rabian Journal for Science and Engineering, vol. 45, no. 3, pp. 2049-2063, 2020.

[26] H. Garg and K. Kumar, "A novel possibility measure to interval-valued intuitionistic fuzzy set using connection number of set pair analysis and its applications," Neural Computing and Applications, vol. 32, no. 8, pp. 3337-3348, 2020.

[27] Y. Liu, J. Liu, Y. Qin, and Y. Xu, "A novel method based on extended uncertain 2-tuple linguistic Muirhead mean operators to MAGDM under uncertain 2-tuple linguistic environment," International Journal of Computational Intelligence Systems, vol. 12, no. 2, pp. 498-512, 2019.

[28] Y. Liu, Y. Qin, L. Xu, H.-B. Liu, and J. Liu, "Multiattribute group decision-making approach with linguistic pythagorean fuzzy information," IEEE Access, vol. 7, pp. 143412-143430, 2019.

[29] H.-C. Liu, M.-Y. Quan, Z. Li, and Z.-L. Wang, "A new integrated MCDM model for sustainable supplier selection under interval-valued intuitionistic uncertain linguistic environment," Information Sciences, vol. 486, pp. 254-270, 2019.

[30] J. Tang, F. Meng, F. J. Cabrerizo, and E. Herrera-Viedma, “A procedure for group decision making with interval-valued intuitionistic linguistic fuzzy preference relations," Fuzzy Optimization and Decision Making, vol. 18, no. 4, pp. 493-527, 2019.

[31] P. Liu and S. M. Chen, "Group decision making based on Heronian aggregation operators of intuitionistic fuzzy numbers," IEEE Transactions on Cybernetics, vol. 47, no. 9, pp. 2514-2530, 2016.

[32] B. Han, H. Chen, J. Zhu et al., "An approach to linguistic multiple attribute decision-making based on unbalanced linguistic generalized heronian mean aggregation operator," Computational Intelligence and Neuroscience, vol. 2018, Article ID 1404067, 2018.

[33] P. Liu and S.-M. Chen, "Multiattribute group decision making based on intuitionistic 2-tuple linguistic information," Information Sciences, vol. 430-431, pp. 599-619, 2018.

[34] C. Tan and X. Chen, "Generalized archimedean intuitionistic fuzzy averaging aggregation operators and their application to multicriteria decision-making," International Journal of Information Technology \& Decision Making, vol. 15, no. 2, pp. 311-352, 2016.

[35] R. B. Nelsen, An Introduction to Copula, Springer Science Business Media, Berlin, Germany, 2013.

[36] Z. Tao, B. Han, and H. Chen, "On intuitionistic fuzzy copula aggregation operators in multiple- attribute decision making," Cognitive Computation, vol. 10, no. 4, pp. 610-624, 2018.

[37] Z. Tao, B. Han, L. Zhou, and H. Chen, "The novel computational model of unbalanced linguistic variables based on Archimedean Copula," International Journal of Uncertainty, Fuzziness and Knowledge-Based Systems, vol. 26, no. 4, pp. 601-631, 2018.

[38] T. Chen, S.-S. He, J.-Q. Wang, L. Li, and H. Luo, "Novel operations for linguistic neutrosophic sets on the basis of Archimedean copulas and co-copulas and their application in multi-criteria decision-making problems," Journal of Intelligent \& Fuzzy Systems, vol. 37, no. 2, pp. 2887-2912, 2019.

[39] L. Xu, L. Yi, and H. Liu, "Linguistic interval-valued intuitionistic fuzzy copula power aggregation operators for multiattribute group Decision Making," Journal of Intelligent \& Fuzzy Systems, pp. 1-20. In press, 2020.

[40] C. Bonferroni, "Sulle medie multiple di potenze," Bollettino dell'Unione Matematica Italiana, vol. 5, no. 3-4, pp. 267-270, 1950.

[41] R. R. Yager, "On generalized Bonferroni mean operators for multi-criteria aggregation," International Journal of Approximate Reasoning, vol. 50, no. 8, pp. 1279-1286, 2009.

[42] S. Sykora, Mathematical Means and Averages: GeneralizedHeronian Means, Sykora S, Stan's Library, Italy, 2009.

[43] D. Yu, "Intuitionistic fuzzy geometric Heronian mean aggregation operators," Applied Soft Computing, vol. 13, no. 2, pp. 1235-1246, 2013.

[44] D. J. Yu and Y. Y. Wu, "Interval-valued intuitionistic fuzzy Heronian mean operators and their application in multi- 
criteria decision making," African Journal of BusinessManagement, vol. 6, no. 11, pp. 4158-4168, 2012.

[45] P. Liu, Z. Liu, and X. Zhang, "Some intuitionistic uncertain linguistic Heronian mean operators and their application to group decision making," Applied Mathematics and Computation, vol. 230, pp. 570-586, 2014.

[46] P. Liu, J. Liu, and J. M. Merigó, "Partitioned Heronian means based on linguistic intuitionistic fuzzy numbers for dealing with multi-attribute group decision making," Applied Soft Computing, vol. 62, pp. 395-422, 2018.

[47] G. Zhang, Z. Zhang, and H. Kong, "Some normal intuitionistic fuzzy Heronian mean operators using hamacher operation and their application," Symmetry, vol. 10, no. 6, p. 199, 2018.

[48] H. Zhang, R. Zhang, H. Huang, and J. Wang, "Some picture fuzzy Dombi Heronian mean operators with their application to multi-attribute decision-making," Symmetry, vol. 10, no. 11, p. 593, 2018.

[49] C. Genest and R. J. Mackay, "Copules archimédiennes et families de lois bidimensionnelles dont les marges sont données," Canadian Journal of Statistics, vol. 14, no. 2, pp. 145-159, 1986.

[50] P. Liu, "Multiple attribute group decision making method based on interval-valued intuitionistic fuzzy power Heronian aggregation operators," Computers \& Industrial Engineering, vol. 108, pp. 199-212, 2017.

[51] Y. D. He, H. Y. Chen, L. G. Zhou, J. P. Liu, and Z. F. Tao, "Generalized interval-valued atanassov's intuitionistic fuzzy power operators and their application to group decision making," International Journal of Fuzzy Systems, vol. 15, pp. 401-411, 2013.

[52] H. Zhang, "Linguistic intuitionistic fuzzy sets and application in MAGDM," Journal of Applied Mathematics, vol. 2014, Article ID 432092, 11 pages, 2014.

[53] P. Liu and P. Wang, "Some improved linguistic intuitionistic fuzzy aggregation operators and their applications to multiple-attribute decision making," International Journal of Information Technology \& Decision Making, vol. 16, no. 03, pp. 817-850, 2017. 